ARTICLE

\title{
Cellular and molecular landscape of mammalian sinoatrial node revealed by single-cell RNA sequencing
}

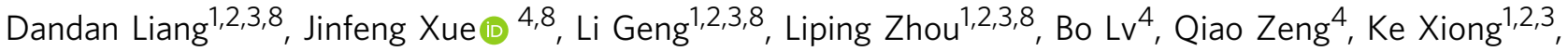

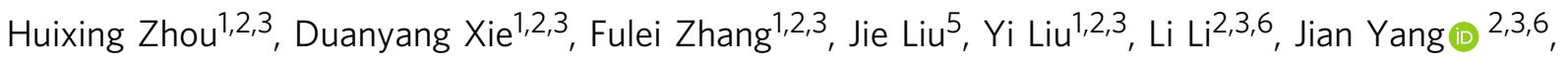 \\ Zhigang Xue (1) ${ }^{4,7 凶}$ \& Yi-Han Chen (1) 1,2,3,6凶
}

Bioelectrical impulses intrinsically generated within the sinoatrial node (SAN) trigger the contraction of the heart in mammals. Though discovered over a century ago, the molecular and cellular features of the SAN that underpin its critical function in the heart are uncharted territory. Here, we identify four distinct transcriptional clusters by single-cell RNA sequencing in the mouse SAN. Functional analysis of differentially expressed genes identifies a core cell cluster enriched in the electrogenic genes. The similar cellular features are also observed in the SAN from both rabbit and cynomolgus monkey. Notably, Vsnl7, a core cell cluster marker in mouse, is abundantly expressed in SAN, but is barely detectable in atrium or ventricle, suggesting that Vsnl1 is a potential SAN marker. Importantly, deficiency of Vsnl1 not only reduces the beating rate of human induced pluripotent stem cell - derived cardiomyocytes (hiPSC-CMs) but also the heart rate of mice. Furthermore, weighted gene co-expression network analysis (WGCNA) unveiled the core gene regulation network governing the function of the SAN in mice. Overall, these findings reveal the whole transcriptome profiling of the SAN at single-cell resolution, representing an advance toward understanding of both the biology and the pathology of SAN.

\footnotetext{
${ }^{1}$ Department of Cardiology, Shanghai East Hospital, Tongji University School of Medicine, Shanghai 200120, China. ${ }^{2}$ Key Laboratory of Arrhythmias of the Ministry of Education of China, Shanghai East Hospital, Tongji University School of Medicine, Shanghai 200120, China. ${ }^{3}$ Institute of Medical Genetics, Tongji University, Shanghai 200092, China. ${ }^{4}$ Department of Regenerative Medicine, Tongji University School of Medicine, Shanghai 200092, China. ${ }^{5}$ Translational Center of Stem Cell Research, Tongji Hospital, Tongji University School of Medicine, Shanghai 200065, China. ${ }^{6}$ Department of Pathology and

Pathophysiology, Tongji University School of Medicine, Shanghai 200092, China. ${ }^{7}$ Reproductive Medicine Center, Tongji Hospital, Tongji University School of Medicine, Shanghai 200065, China. ${ }^{8}$ These authors contributed equally: Dandan Liang, Jinfeng Xue, Li Geng, Liping Zhou. ${ }^{凶}$ email: xuezg@tongji.edu.cn; chenyh@cashq.ac.cn
} 
T he sinoatrial node (SAN) is the pacemaker of the heart in mammals. The spontaneous bioelectrical activity of a relatively small population of pacemaker cells in SAN initiates the heart beat and controls heart rates ${ }^{1-3}$. The SAN is a small, specialized and heterogeneous myocardial structure located at the junction of the superior vena cava and right atrium ${ }^{4}$. The pacemaker activity of SAN cells originates from the synergistic effect of ion channels, transporters, and $\mathrm{Ca}^{2+}$ regulators ${ }^{3,5,6}$. In addition, it is tightly regulated by autonomic nerve signaling ${ }^{7}$.

Both histologically and functionally, SAN presents significant heterogeneity. In terms of cell morphology, cells in different regions of SAN show a variety of shapes. It has been observed clinically for a long time that there is a "leading pacemaker" site within the SAN, and the "leading pacemaker" site can drift around in SAN, suggesting that bioelectrical activity of SAN pacemaker cells is not uniform ${ }^{8-10}$. There is an urgent need to reveal systematically the cellular taxonomic basis and molecular regulatory networks that make up these heterogeneities.

Recently, Linscheid et al. ${ }^{11}$ systematically analyzed the proteomics of SAN biopsies in mice, and then linked the measured protein abundances to specific cell types by performing SAN single-nucleus RNA sequencing (snRNA-seq). However, the characteristic and regulatory network of SAN pacemaker cells, the key cells generating spontaneous rhythm, need further investigation. With the advantage of single-cell RNA sequencing (scRNA-seq) more expressed genes can be detected and used for comprehensive analysis of cell subtype and regulatory network at transcriptome level. Now we are more likely to identify cellular heterogeneity and explore differential gene expression patterns in a tiny and complex tissue like the SAN ${ }^{12}$. Properties of SAN in adult mammals, such as low cell number and large cell size, have restricted analysis of its whole transcriptomics characteristics at single-cell resolution by high-throughput methods. We obtained SAN single cells from different mammalian species by manual cell picking, then proceeded scRNA-seq to depict the gene expression pattern of SAN.

In the present study, we demonstrated the molecular panorama of SAN cell clusters and the core molecular regulation network underlying the SAN pacemaker activity. Our study classified mouse SAN cells into four clusters, including one core cell cluster, and similar cellular features were also observed in SAN from both rabbit and cynomolgus monkey. We identified a marker Vsnll, which was highly and specifically expressed in the mouse SAN but rarely detected in the atrium or ventricle. Functional studies showed that Vsnll played a pivotal role in regulating the frequency of electrical activity in mouse SAN. Moreover, the consistency of its abundant expression in SAN across species makes it a possible candidate marker gene in other two species as well.

\section{Results}

Distinct clusters of SAN cells in adult mouse. We isolated and pooled 771 cells, including 718 cells from microdissected SAN tissues of 21 adult mice and 53 atrial and ventricular (AV) cardiomyocytes from 4 adult mice, then performed scRNA-seq using the Smart-seq 2 protocol ${ }^{12,13}$ (Fig. 1a and Supplementary Fig. 1) to examine their transcriptomes. We achieved an average of nine million reads per cell and an average of 7586 genes per cell was detected. The gene expression was quantified using trimmed clean reads. Clustering analysis of cells was determined based on the expression of 2500 mouse heart-related genes previously reported by Vedantham et al. ${ }^{6}$ (Supplementary Data 1). The $t$ distributed stochastic neighbor embedding ( $\mathrm{t}-\mathrm{SNE}$ ) analysis revealed four distinct, previously undescribed cell clusters within the mouse SAN (Clusters 1-4) and one AV cell cluster (Cluster 5) (Fig. $1 \mathrm{~b}$ and Supplementary Figs. 2 and 3). The distinct cell clusters of SAN were identified based on the functional analysis of differentially expressed genes (DEGs) of each cluster (Fig. 1c and Supplementary Data 2). Cluster 1 highly expressed genes involved in calcium ion transport, regulation of heart rate, and membrane depolarization of SAN cells. In addition to expressing pacemaker cell markers, Cluster 2 and Cluster 3 were enriched in genes for cell adhesion and extracellular matrix organization, respectively. Cluster 4 highly expressed classical markers of pacemaker cells and the basic electrogenic genes involved in SAN pacemaker activity including ion channels, transporters, $\mathrm{Ca}^{2+}$ regulators, and autonomic nerve signaling (Fig. 1d, e). Cluster 1 is similar to Cluster 4 but with relatively lower expression of classic markers and less rhythm related genes. We therefore speculated that Cluster 4 might be the core cell cluster and have more important roles in SAN function compared to other clusters.

DEG analysis showed that Cluster 4 highly expressed known markers for SAN including Hcn4 and Honl (refs. ${ }^{14,15}$ ). Besides ion channels, two genes related to autonomic nervous system regulation, Ednrb and Pde1a, were also highly expressed in this cluster (Fig. 1c, d). Pde1a, a cyclic nucleotide phosphodiesterase which regulates cAMP metabolism, drives SAN pacemaker function and indices of heart rate variability ${ }^{16}$. These cell markers indicated that this population might be the core cell cluster, representing the presumed pacemaker cells with the highest pacing frequency. In addition, Gene Ontology (GO) enrichment analysis of DEGs confirmed that Cluster 4 is associated with the regulation of heart rate (Fig. 1e). Notably, we detected three genes, namely Vsnl1 (a member of the visinin/recoverin subfamily of neuronal calcium sensor proteins), Unc80 (Unc-80 homolog, NALCN channel complex subunit), and Dlgap1 (the disks large-associated protein 1), which were relatively highly and specifically expressed in Cluster 4 compared to other clusters (Fig. 1d), suggesting their utility as potential markers of SAN core cells. Cluster 1 highly expressed genes of calcium ion transport (Ryr3, Doc2g, Dsg2, Fgf12, Abcc8, and Sln); Cluster 2 expressed genes involved in cell adhesion and multiple cell signaling pathways (Apold1, Cd300lg, Cxcl12, Cldn5, and Rgs5); Cluster 3 highly expressed extracellular matrix and collagen markers related genes (Col1a1, Col3a1, Mmp2, and Thbs1) (Fig. 1c, d). GO analysis indicated that the DEGs enriched in each cluster corresponded to their characteristic biological processes (Fig. 1e).

To investigate the expression pattern of these cell cluster marker genes in the heart, we dissected the mouse SAN, atrium, and ventricle tissues and performed qPCR (Figs. 2a and 3a). We found that the expression pattern of Vsnl1, Dlgap1, and Unc80 was similar to that of Hcn4, which is relatively highly expressed in SAN compared to atrium and ventricle (Fig. 2a), though the transcriptional level of Unc80 was lower than that of Vsnll and Dlgap1 in SAN (Supplementary Fig. 4). We then analyzed VSNL1, DLGAP1, and UNC80 protein expression in SAN by immunofluorescence. The results showed that though VSNL1 and DLGAP1 were ubiquitously distributed throughout the SAN, while they exhibited heterogeneous expression in different regions within SAN (Fig. $2 b, c, e, f$ ). Consistent with the mRNA level, the low protein expression of UNC80 was observed in SAN (Fig. 2d, g). Notably, VSNL1 was more specifically expressed than DLGAP1 and UNC80 in SAN, and was almost undetectable in atrium and ventricle (Fig. 2a-d and Supplementary Fig. 5). In addition, other three cell cluster markers, such as RYR3 (Cluster 1), APOLD1 (Cluster 2), and COL1A1 (Cluster 3), were all readily detected in SAN (Fig. 3b-d), demonstrating the reliability of our scRNA-Seq data.

The SAN cell clusters can be segregated from the AV cell cluster. In addition to the mouse SAN cells, we simultaneously 
a

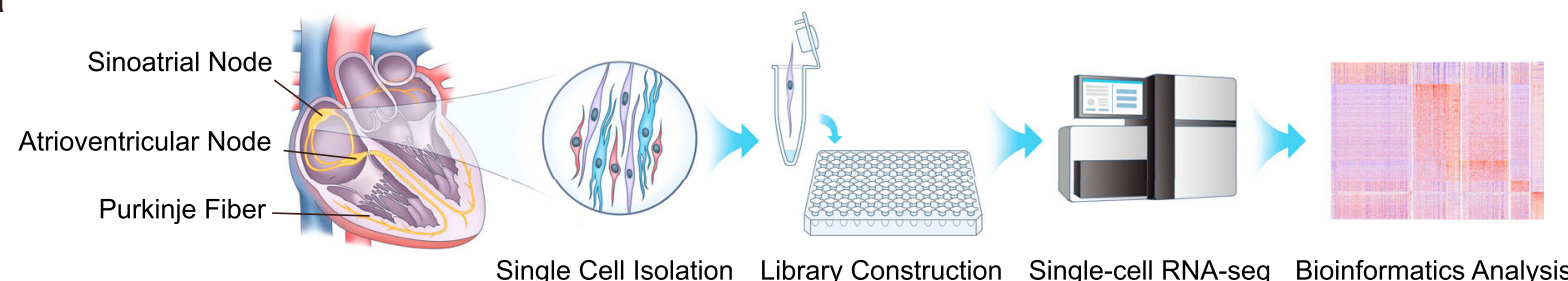

b

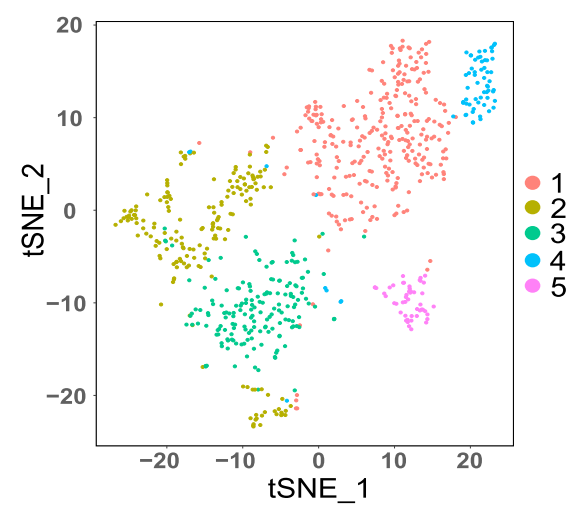

d
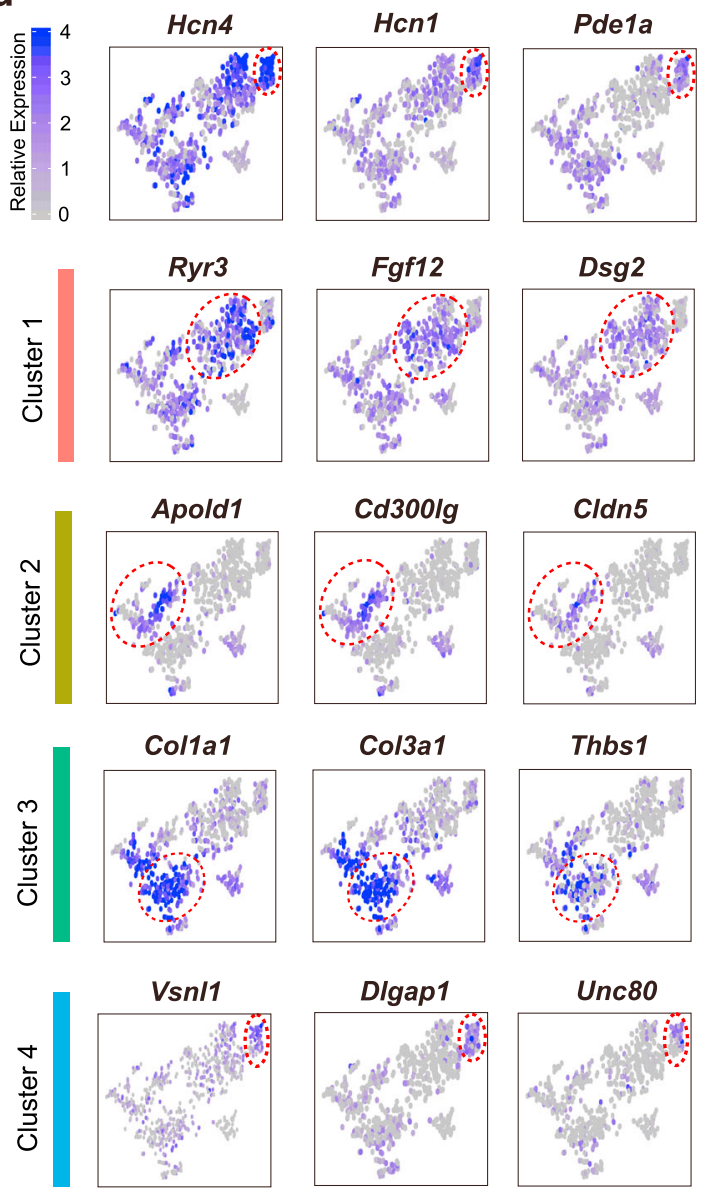

C

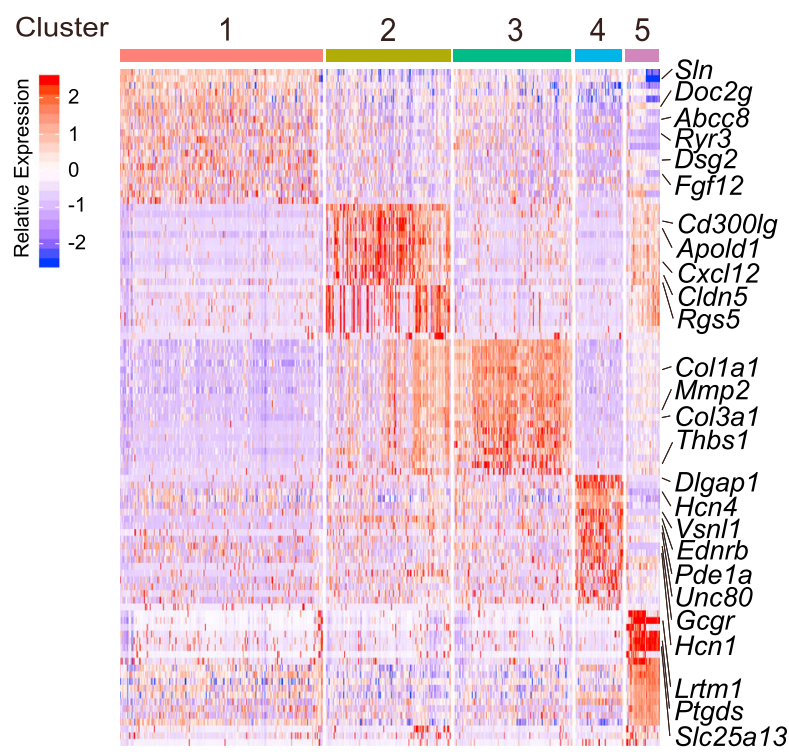

e

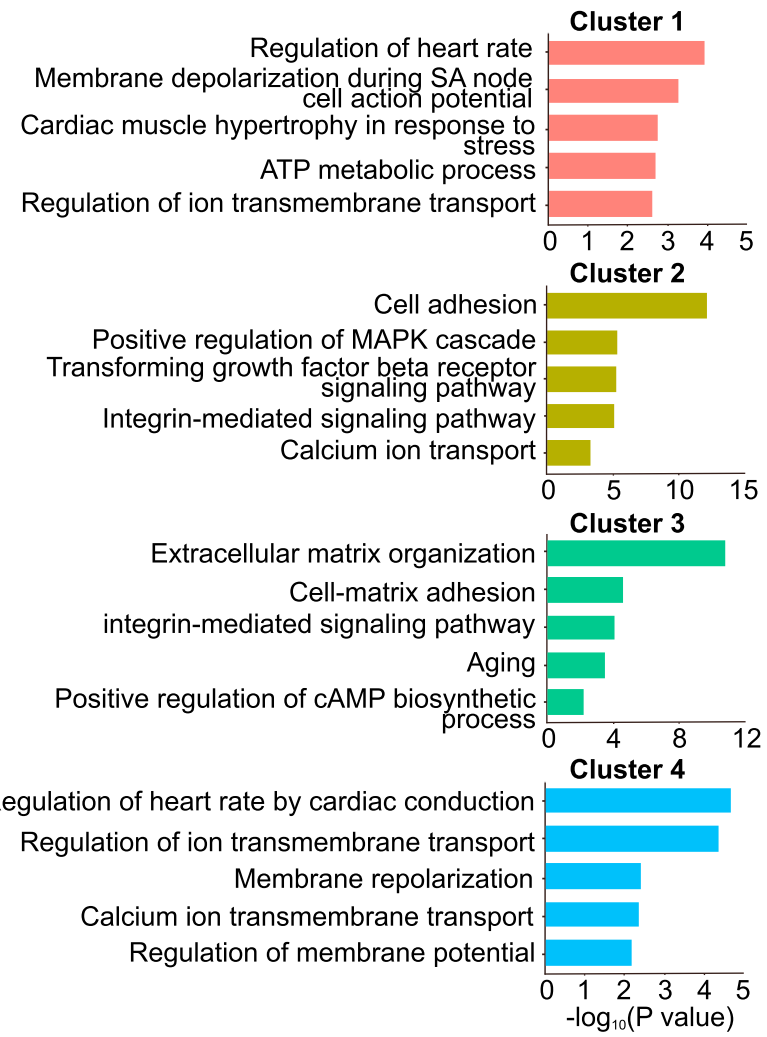

analyzed AV cells, which have no pacemaker activity and were well-segregated based on their transcriptomes into a distinct cluster (Cluster 5) (Fig. 1b and Supplementary Fig. 3a). Moreover, several genes, such as Lrtm1, Ptgds, and Slc25a13, were exclusively and highly expressed in Cluster 5, suggesting their utility as specific markers of AV cells to distinguish them from SAN cells

(Fig. 1c and Supplementary Fig. 3b). We then profiled the expression of distinctive ion channels in Cluster 5 (AV cell cluster) and Cluster 4 (SAN core cell cluster). These ion channel genes were differentially expressed in atrioventricular and SAN tissues according to previous reports ${ }^{11,17,18}$. In general, the gene expression profiles distinguished these two cell clusters as 
Fig. 1 Classification and functional analysis of mouse sinoatrial node (SAN) cells. a Illustrator and workflow for single-cell RNA sequencing (scRNA-seq) of SAN. b $t$-distributed stochastic neighbor embedding (t-SNE) analysis identified five clusters including four SAN cell clusters (Clusters 1, 2, 3, 4) and one atrial and ventricular (AV) cardiomyocytes cell cluster (Cluster 5) ( $n=771$ biologically independent cells of 25 independent animals). c Heat map shows top differentially expressed genes (DEGs) of each cluster. Cluster 4 was highly expressed cell markers including Hcn4, Hcn1, and Ednrb, Pdela and identified as the core cell cluster. d Feature plot of DEGs shows Vsnl1, Unc80, and Dlgap1 were expressed more specifically than Hcn4 and Hcn1 in Cluster 4. Location of each cluster was marked with the red circle. e Gene Ontology (GO) terms show the biological processes of SAN cell clusters and Cluster 4 was mainly associated with regulation of heart rate and ion transmembrane transport, while Cluster 1, 2, and 3 were all related to electrical activity but had certain difference.

expected. We found that Cluster 5 was characterized by higher expression of Gja5 and Gja1, and lower expression of Cacnalg, Cacnalg and the auxiliary $\mathrm{Ca}^{2+}$ channel subunits Cacna2d2 whereas Cluster 4 was characterized by higher expression of $\mathrm{Hcn} 1$ and Hcn4, and lower expression of Gjal. However, we noted that the expression of Cacna1c and Ryr2, which was thought to be slightly lower in SAN tissues than in atria and ventricles ${ }^{18}$, was almost identical in Cluster 5 and Cluster 4. Slc25a13, which is associated with calcium ion binding, was highly and exclusively expressed in Cluster 5 compared to Cluster 4, so were Lrtm1 and Ptgds (Supplementary Fig. 3c).

Vsnl1 is involved in the regulation of SAN function. To explore the possible role of distinct cell clusters in SAN, we interfered several marker genes in each cluster on human induced pluripotent stem cell-derived cardiomyocytes (hiPSC-CMs) to record changes in beating rate with microelectrode array (MEA). Our results showed that $V s n l 1$ deficiency reduced the beating rate of hiPSC-CMs by $44.8 \%$ of the control (Fig. $4 \mathrm{a}, \mathrm{b}$ ), suggesting it is involved in the regulation of cellular autonomic rhythm. However, the knockdown of marker genes in other three clusters did not alter this parameter (Supplementary Fig. 6). To further verify the effects of Vsnl1 on SAN function in vivo, we measured the heart rate in conscious mice after AAV-mediated gene knockdown with AAV9-miRNAi-Vsnl1 or AAV9-miRNAi-Control by the telemetry. In agreement with the MEA data, Vsnll deficiency also reduced the heart rate of the mouse (Fig. 4c, d), validating its role in SAN function.

Next, we analyzed the effects of Vsnll on molecules involved in bioelectrical activity, such as ion channels and $\mathrm{Ca}^{2+}$ regulators expression in both Vsnll knockdown neonatal rat cardiomyocytes and mouse SAN. For the Vsnl1 knockdown neonatal rat cardiomyocytes, at transcription level, the expression of the voltage-dependent $\mathrm{Ca}^{2+}$ channels $\mathrm{Ca}_{\mathrm{v}} 3.3$ (Cacnali) and $\mathrm{Ca}^{2+}$ transporters (Serca2 and Ryr2) was downregulated, whereas there is no statistical difference in the expression of other channels, including voltage-dependent $\mathrm{K}^{+}$channels (Kcna4, Kcnh2, Kcnj3, Kcnj5, and Kcnj11), HCN channels (Hcn4), voltage-gated $\mathrm{Na}^{+}$ channels $\mathrm{Na}_{\mathrm{v}} 1.5$ (Scn5a), voltage-dependent $\mathrm{Ca}^{2+}$ channels $\mathrm{Ca}_{\mathrm{v}} 1.2$ and $\mathrm{Ca}_{\mathrm{v}} 1.3$ (Cacnalc and Cacna1d), and $\mathrm{Ca}^{2+}{ }_{\text {-regulators }}$ (Ncxl) (Supplementary Fig. 7). We specifically extracted SAN regions of AAV9-miRNAi-Vsnl1 and AAV9-miRNAi-Control mouse to determine the interference efficiency of Vsnll and analyze these gene expression. The expression of Vsnl1 significantly reduced in the SAN of AAV9-miRNAi-Vsnl1 mouse (Supplementary Fig. 8). In AAV9-miRNAi-Vsnl1 SAN, besides Hcn4, the transcriptomic expression of Cacnald, Cacnali, and Serca2a were significantly downregulated, with no statistical changes in the expression of Kcna4, Kcnh2, Kcnj3, Kcnj5, Kcnj11, Scn5a, Cacnalc, Ryr2, and Ncx1 (Supplementary Fig. 8). The knockdown of VSNL1 and HCN4 has also been shown in AAV9miRNA-Vsnl1 SAN section with VSNL1 and HCN4 antibody costaining (Supplementary Fig. 9), consistent with the decreased mRNA expression of Vsnll and Hcn4 in AAV9-miRNA-Vsnl1 SAN (Supplementary Fig. 8).
Gene expression network of SAN cells in adult mouse. To investigate gene expression programs governing SAN pacemaker activity, weighted gene co-expression network analysis (WGCNA) was performed to identify distinct modules of gene expression in the scRNA-seq data (Fig. 5a). We identified ten coexpression modules, six out of which were related to the regulation of ion channels. Notably, we found that two modules (red and brown) were mainly enriched in GO terms of heart rate, cardiac conduction, membrane potential, and ion transport (Fig. 5b). These two modules were comprised of three different classes of key genes associated with heart rhythm (Supplementary Data 3). The first class is ion channel genes, such as Hcn4 and Cacnald. Hcn4 encodes the hyperpolarization-activated, cyclic nucleotide-gated channel responsible for the hyperpolarizationactivated or funny current $\left(I_{\mathrm{f}}\right)$, which has a key role in the pacemaker potential of $\mathrm{SAN}^{18,19}$. Cacnald encodes the voltagegated L-type $\mathrm{Ca}^{2+}$ channel $\mathrm{Ca}_{\mathrm{v}} 1.3$, which activates during the diastolic depolarization phase of SAN pacemaker cells and may constitute the predominant voltage-dependent mechanism contributing to pacemaking ${ }^{2,20}$. Loss-of-function of $\mathrm{Ca}_{\mathrm{v}} 1.3$ in both mouse and humans causes sick sinus syndrome characterized by severe bradycardia ${ }^{21-23}$. The second class is transporter genes, such as Slc8a1 and Atp1a1. Slc8a1 encodes $\mathrm{Na}^{+} / \mathrm{Ca}^{2+}$ exchange 1 (NCX1), which is a major actor in cardiac cell $\mathrm{Ca}^{2+}$ homeostasis and is responsible for $\mathrm{Ca}^{2+}$ efflux during action potential repolarization $^{2,24}$. Intact NCX1 activity is necessary for maintaining automaticity of pacemaker cells ${ }^{25,26}$. Atpla1 encodes $\mathrm{Na}^{+} / \mathrm{K}^{+}$ pump $\left(\mathrm{Na}^{+} / \mathrm{K}^{+}\right.$-ATPase), which acts as a transporter to maintain intracellular ion homeostasis and generates a net outward current that influences cellular pacemaking 27,28 . The third class is $\mathrm{Ca}^{2+}$ regulator genes, such as Ryr2 and Camk2a. Ryr2 encodes the intracellular $\mathrm{Ca}^{2+}$ channels ryanodine receptor 2 (RYR2), activated by $\mathrm{Ca}^{2+}$ influx due to subsarcolemmal local $\mathrm{Ca}^{2+}$-induced $\mathrm{Ca}^{2+}$ release (LCICR), which controls chronotropic state of SAN pacemaker cells and is the predominant mechanism of SAN automaticity " $\mathrm{Ca}^{2+}$ clock" hypothesis ${ }^{2}$. Camk2a encoding $\mathrm{Ca}^{2+}$ calmodulin-dependent protein kinase II (CaMKII) is a multifunctional serine/threonine kinase targeting a large number of substrates, including ion channels, pumps, $\mathrm{Ca}^{2+}$ cycling proteins, and transcription factors. In SAN, CaMKII controls pacemaker cell activity by regulating the activation and reactivation kinetics of L-type $\mathrm{Ca}^{2+}$ channels, thus affecting pacemaker cell activity ${ }^{2,29}$. Moreover, autonomic nerve signaling/G protein-coupled receptor genes (Gnao1, $R x f p 1$, and $G c g r$ ) and transcription factor genes (Shox2, Tbx3 and Bmp2) were identified in these two modules as well. In general, the co-expression network of the red module mainly contains $\mathrm{K}^{+}$channels and $\mathrm{Ca}^{2+}$ channels genes (Fig. 5c), while the brown module tends to contain more $\mathrm{Ca}^{2+}$ regulator genes (Fig. $5 \mathrm{~d}$ ).

Predicated protein-protein interaction of the red and brown modules reflected the similar biological processes with GO analysis, and the construction of interaction network showed the connectivity of the ion channels, transporters, $\mathrm{Ca}^{2+}$ regulators, autonomic nerve signaling/G protein-coupled receptors, and transcription factors in these two critical modules 
a

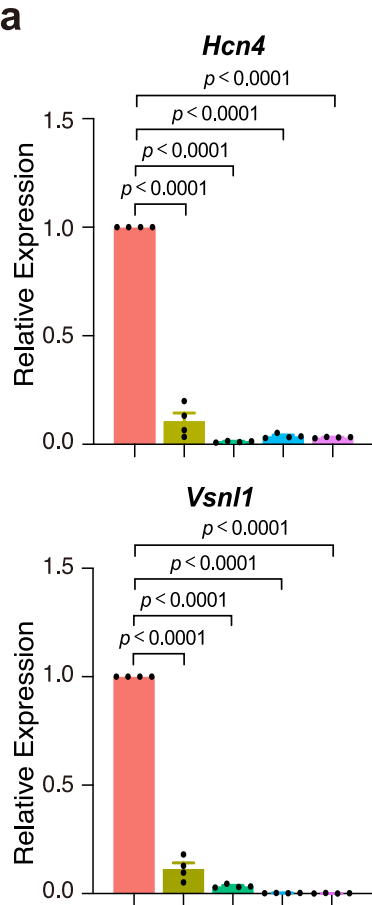

b

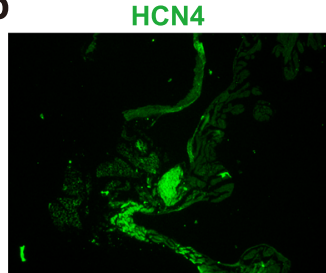

c

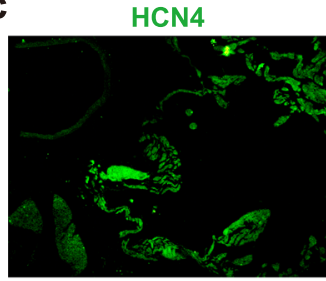

d

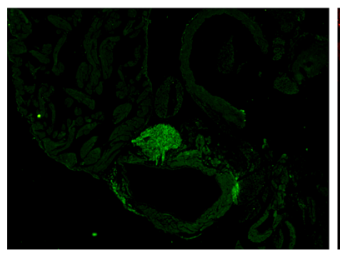

VSNL1

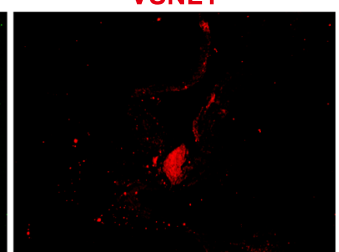

DLGAP1

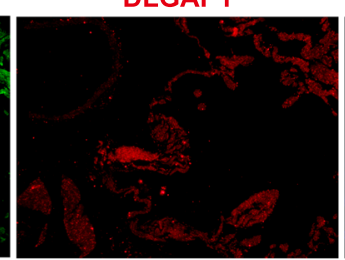

UNC80
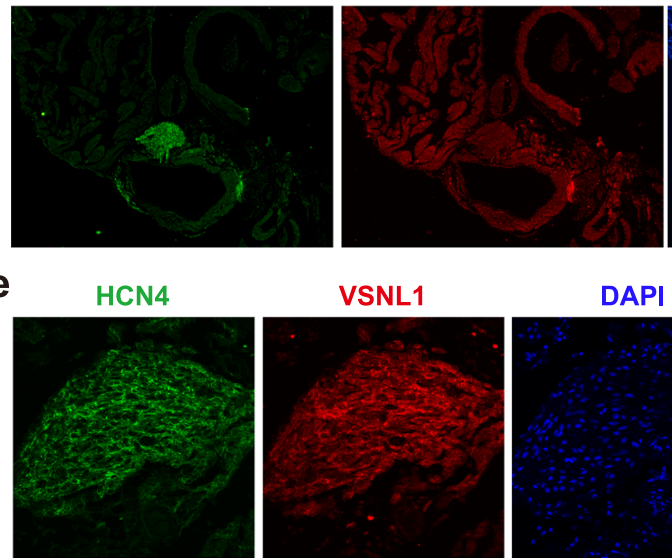

HCN4

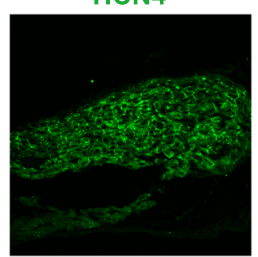

g

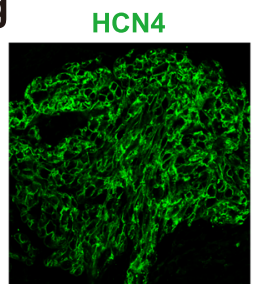

VSNL1

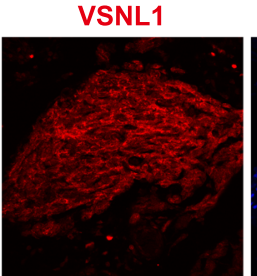

DLGAP1

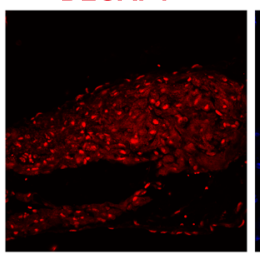

UNC80

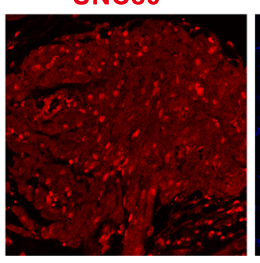

DAPI

DAPI

DAPI
DAPI

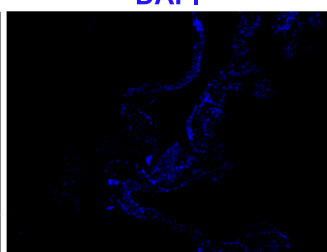

DAPI

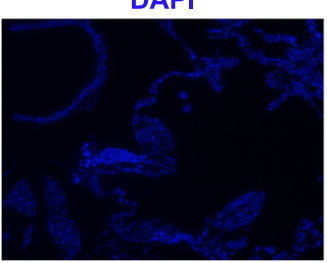

DAPI
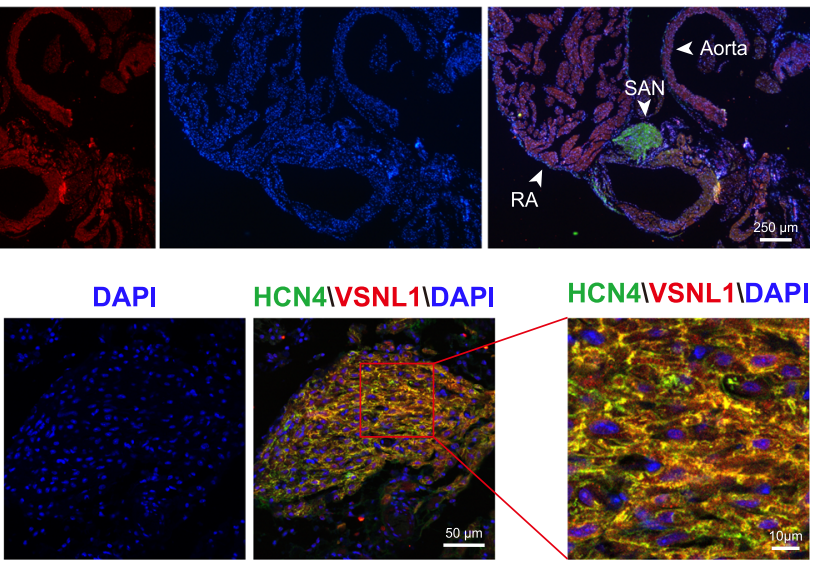

HCN4IVSNL1IDAP

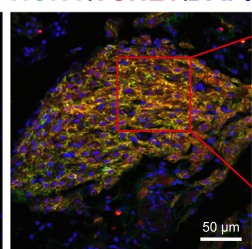

HCN4IVSNL1IDAPI
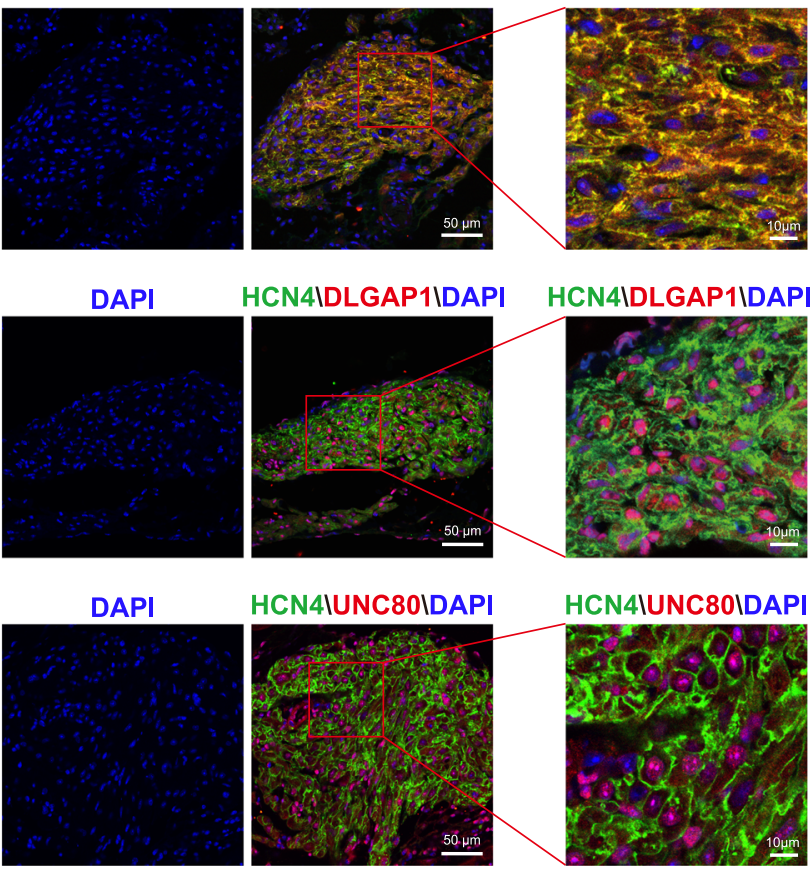

Fig. 2 Expression pattern of the core cluster markers Vsnl1, Dlgap1, and Unc80 in mouse heart. a qPCR analysis shows the expression of Hcn4, Vsnl1, Dlgap1, and Unc80 in sinoatrial node (SAN), atrial, and ventricular tissue, respectively ( $n=4$ biologically independent animals per group), Dunnett's multiple comparisons test, data are represented as mean \pm s.e.m., adjusted $p$ value was labeled on the top. RA right atrium, LA left atrium, RV right ventricle, LV left ventricle. b-g Immunohistochemical analysis of VSNL1, DLGAP1, and UNC80 in SAN slices at low magnification. Representative images are shown from $n=3$ biologically independent samples. b Immunofluorescence images of HCN4 (green), VSNL1 (red), and DAPI (blue). c Immunofluorescence images of HCN4 (green), DLGAP1 (red), and DAPI (blue). d Immunofluorescence images of HCN4 (green), UNC80 (red), and DAPI (blue). b-d The arrows indicate the tissues surrounding the SAN. Scale bar $=250 \mu \mathrm{m}$. e-g Localization of the VSNL1, DLGAP1, and UNC80 within SAN at high magnification. e Immunofluorescence images of HCN4 (green), VSNL1 (red), and DAPI (blue). f Immunofluorescence images of HCN4 (green), DLGAP1 (red), and DAPI (blue). $\mathbf{g}$ Immunofluorescence images of HCN4 (green), UNC80 (red), and DAPI (blue). Scale bar = 50 $\mu$ m. e-g Zoom images (the red box regions in the merged image) show the higher magnification. Scale bar $=10 \mu \mathrm{m}$. 
a

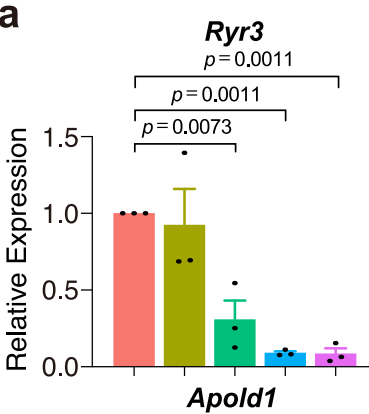

b
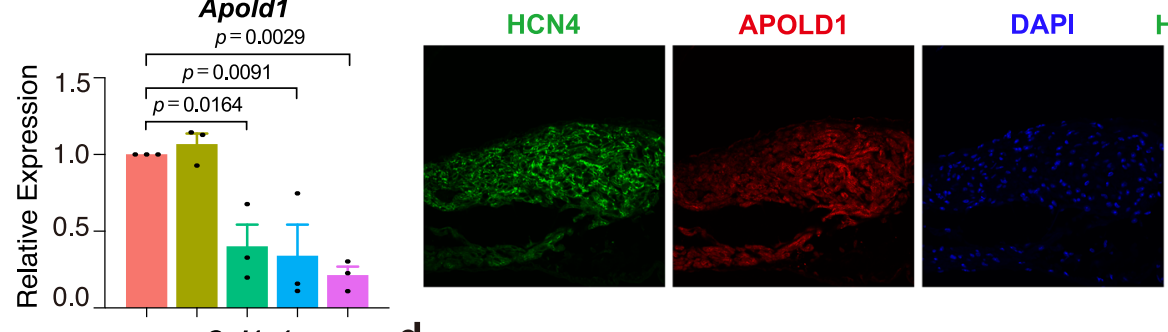

HCN4IAPOLD1IDAPI
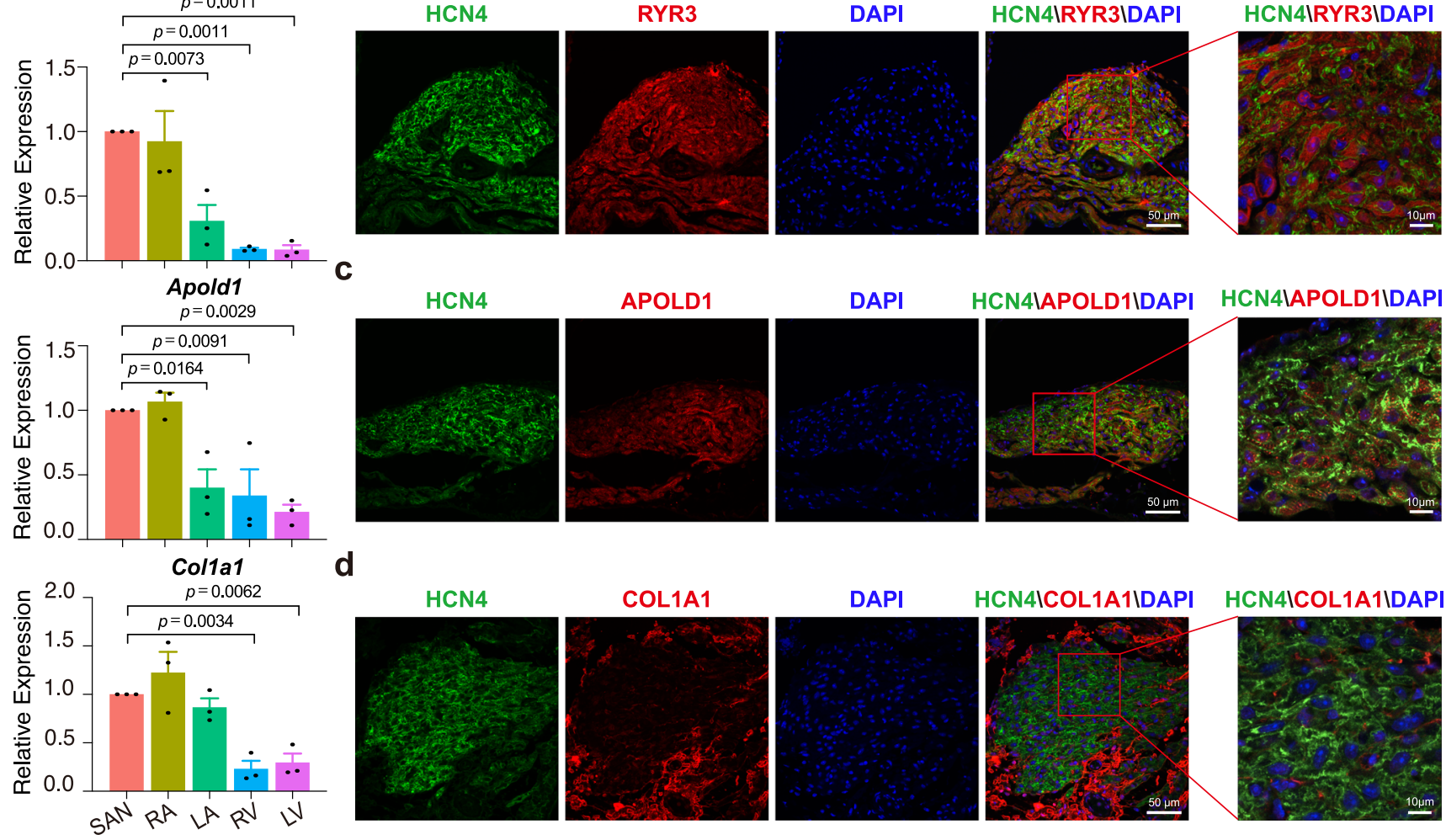

d
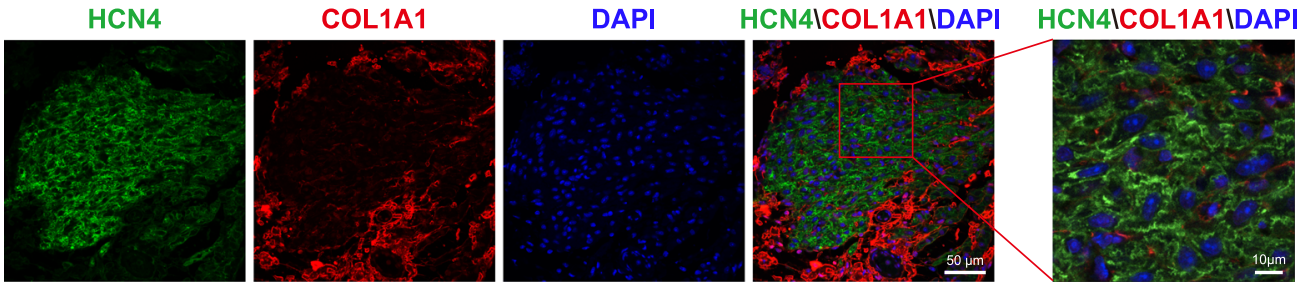

Fig. 3 The expression pattern of non-core cluster genes Ryr3, Apold1, and Col1a1 in mouse heart. a qPCR shows the expression of Ryr3 (Cluster 1), Apold1 (Cluster 2), and Colla1 (Cluster 3) in sinoatrial node (SAN), atrial, and ventricular tissue, respectively ( $n=3$ biologically independent animals per group). Dunnett's multiple comparisons test, data are represented as mean \pm s.e.m., adjusted $p$ value was labeled on the top. RA right atrium, LA left atrium, RV right ventricle, LV, left ventricle. b-d Immunostaining shows the location of RYR3 (b), APOLD1 (c), and COL1A1 (d) in mouse SAN tissue, respectively. Representative images are shown from $n=3$ biologically independent samples. Scale bar $=50 \mu \mathrm{m}$. Zoom images (the box regions in the merged images) show the higher magnification. Scale bar $=10 \mu \mathrm{m}$.

(Supplementary Fig. 10a, b). Interestingly, we found that the core cell cluster gene Gcgr (encoding the glucagon receptor, GCGR), which was involved in GPCR signaling and cAMP biosynthetic process through interaction with $R x f p 1$ and Adcy5 (Fig. 1c and Supplementary Fig. 10a, c), was also identified as a hub gene in the co-expression network of the red module (Fig. 5c). Other related modules, including the blue, yellow, pink, and turquoise modules, possessed a high correlation coefficient and represented genes involved in cell adhesion, extracellular matrix organization, and cell signaling pathways (Supplementary Fig. 10d).

Distinct clusters and gene expression network of SAN cells in rabbits. We next set to compare the SAN gene expression patterns between mouse and rabbit. We isolated 343 SAN cells from six adult rabbits and performed scRNA-seq as described above to detect cell clusters of gene expression. The distinct cell clusters of rabbit SAN were identified mainly using the same criteria as described in mouse. Among three clusters found in rabbit SAN cells (Fig. 6a and Supplementary Fig. 11), Cluster 2 highly expressed ion channel and transporter genes (HCN4, HCN1, ATP1A1, and SLC8A1), $\mathrm{Ca}^{2+}$ regulator genes (ATP2A2, RYR2, CXADR, CASQ2, TRDN, and $A N K R D 1)$, and the angiotensin II receptor (AGTR1) which are of functional relevance to SAN pacemaker activity, thus cluster 2 was defined as the core cluster in rabbit SAN. Meanwhile, Cluster 1 highly expressed genes of the androgen receptor $(A R), \mathrm{Ca}^{2+}$-binding proteins ( $L P L$ and $L U M)$, extracellular matrix (COL1A2 and $M M P 2)$, and ion channels. Cluster 3 highly expressed genes of cardiac gap junction genes (KCNJ8, KCNE4, and GJA1), autonomic nerve signaling/G protein-coupled receptor genes (S1PR1 and
$E D N R B)$, and cell adhesion genes (SELE and CD1D), respectively (Fig. 6b-d, Supplementary Fig. 11 and Supplementary Data 4). GO enrichment analysis demonstrated that Cluster 2 was mainly related to regulation of heart rate by cardiac conduction; meanwhile, the biological function of Clusters 1 and 3 were also confirmed by DEG analysis in each cluster (Fig. 6e). Interestingly, CXADR, encoding the coxsackie and adenovirus receptor which is predominantly expressed at the intercalated discs of cardiomyocytes ${ }^{30,31}$, was highly expressed in Cluster 2 (Fig. 6c). Though the role of CXADR in SAN has not been demonstrated, we speculate that it may be involved in the conduction between SAN cells. We further investigated the transcriptional level of the core cell cluster markers in different regions of rabbit heart, the results showed that these genes (HCN4, HCN1, CASQ2, TRDN, AGTR1, CXADR) were expressed relatively specific in the SAN (Supplementary Fig. 12). Notably, VSNL1, the core cell cluster marker in mouse, was also highly and specifically expressed in the rabbit SAN (Supplementary Fig. 13).

WGCNA analysis identified three modules of gene coexpression in rabbit SAN (Supplementary Fig. 14a). The turquoise module contains key genes encoding ion channels, transporters, $\mathrm{Ca}^{2+}$ regulators, autonomic nerve signaling/G protein-coupled receptors and transcription factors, including HCN4, HCN1, KCNH2, CACNA1C, CACNB2, ATP1A1, ATP1B1, SLC8A1, ATP2A2, RYR2, PLN, CASQ2, TRDN, AGTR1, EDNRA, $B M P 2$, and FOXP2 (Supplementary Data 5). Transporters and $\mathrm{Ca}^{2+}$ regulator genes constructed a co-expression network (Supplementary Fig. 14b). Protein interaction analysis verified the key role of the turquoise module in SAN (Supplementary Fig. 14c). 
a
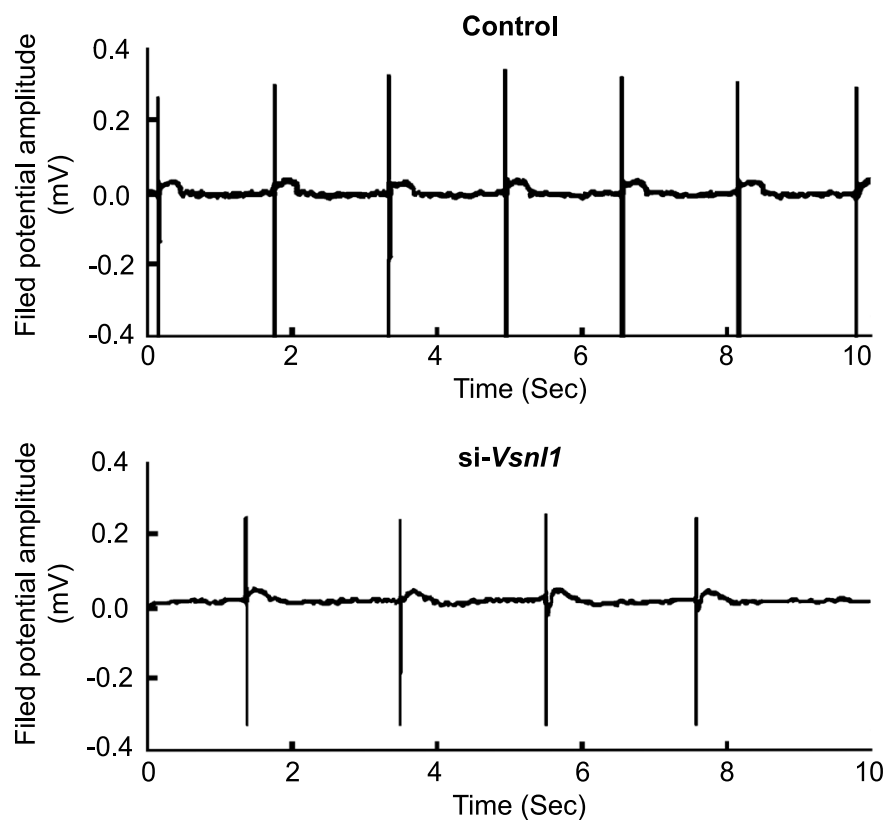

b

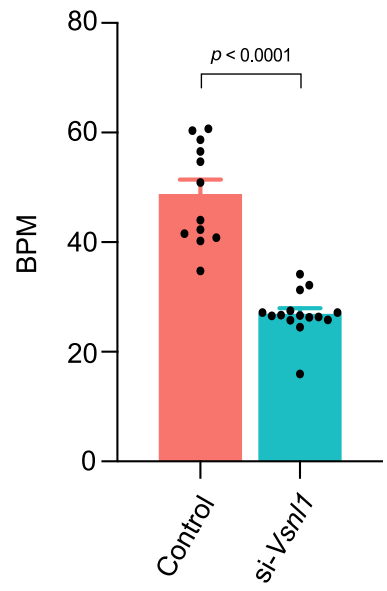

C

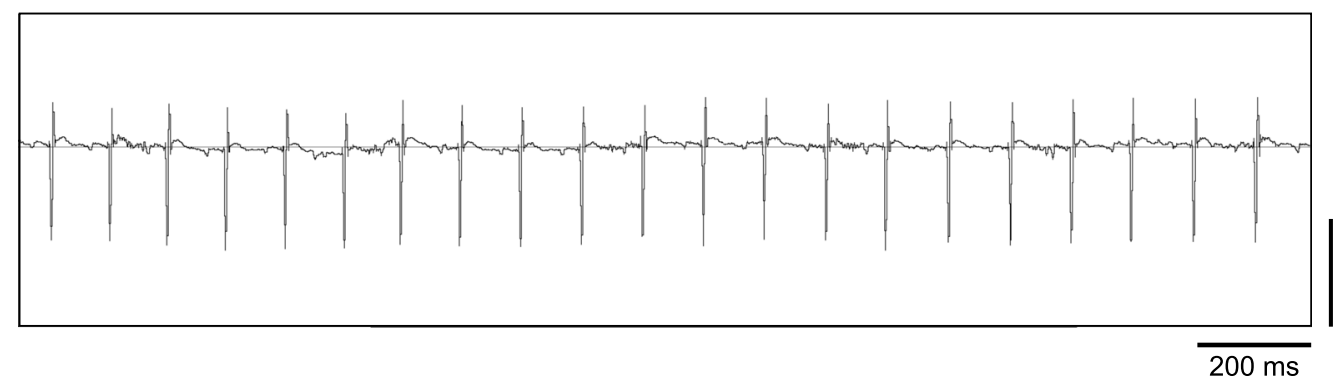

AAV9-miRNAi-Vsnl1

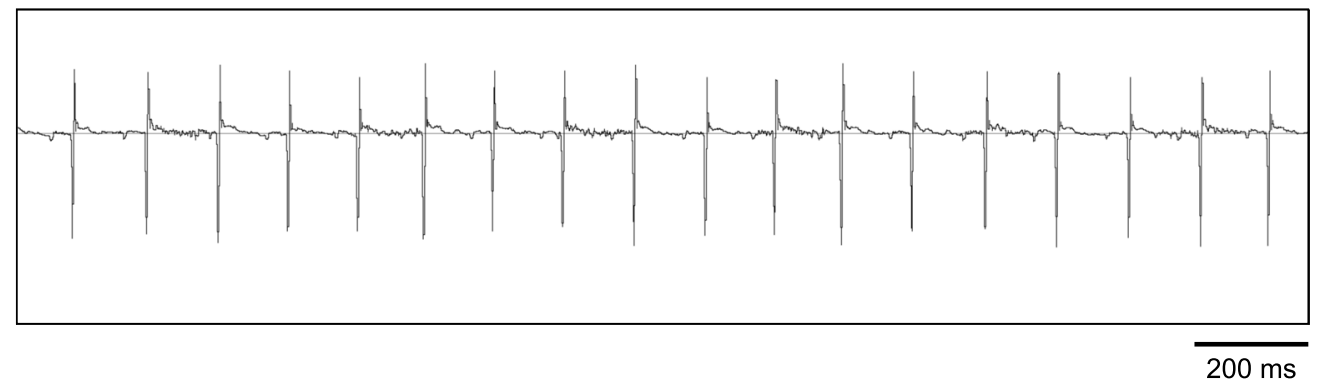

d

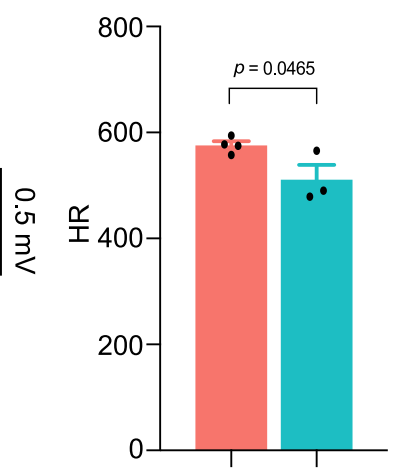

웅

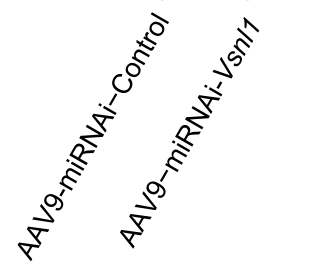

Fig. 4 The core cell cluster marker Vsnl1 governs the pacing activity. a, b Vsnl1 deficiency reduced the beating rate of human induced pluripotent stem cell-derived cardiomyocytes (hiPSC-CMs). a Representative field potential traces in hiPSC-CMs transfected with nonsense siRNA (Control) or Vsn/1 targeting (si-Vsn/1) siRNA. b Statistic data of beating rates. Control: $n=12$ independent examinations over three independent experiments; si-Vsn/1: $n=15$ independent examinations over three independent experiments. BPM beats per minute. c, $\mathbf{d} V s n / 1$ deficiency reduced the heart rate of mouse. $\mathbf{c}$ Representative electrocardiographs (ECGs) obtained from conscious mice through telemetric recording system. $\mathbf{d}$ Statistic data of mouse heart rate (HR). AAV9-miRNAi-Control: $n=4$ biologically independent animals; AAV9-miRNAi-Vsnl1: $n=3$ biologically independent animals. Unpaired, two-tailed Student's $t$-test, data are represented as mean \pm s.e.m., $p$ value was labeled on the top $(\mathbf{b}, \mathbf{d})$.

Distinct clusters of SAN cells in adult cynomolgus monkey. Then we isolated 296 SAN cells from 12 adult cynomolgus monkey and performed transcriptional clustering analysis as described in mouse and rabbit. The SAN cells of the cynomolgus monkeys were segregated into three clusters (Fig. 7a), and functional analysis of DEGs indicated that Cluster 3 was the core cell cluster based on high expression of ion channelrelated genes (ATP1B1, VSNL1, CAMK2D, TCAP, and FXYD6) and autonomic nervous system regulation genes $(A D R B 1$ and
CPT1B). VSNL1, which was highly and specifically expressed in mouse and rabbit SAN, was also the TOP DEGs in Cluster 3, further verified that this cluster is the core cell cluster in monkey. Besides Cluster 3, Cluster 1 was defined by high expression of genes of the $\mathrm{Ca}^{2+}$ regulator (CALD1 and $C A L M 2), G$ protein-coupled receptor signaling molecular $(R H O B)$ and cytoskeletal protein (TAGLN and ACTB), and ion channel, Cluster 2 highly expressed cardiac gap junction genes (ATB1B3 and GJA1), autonomic nerve signaling/G 
a
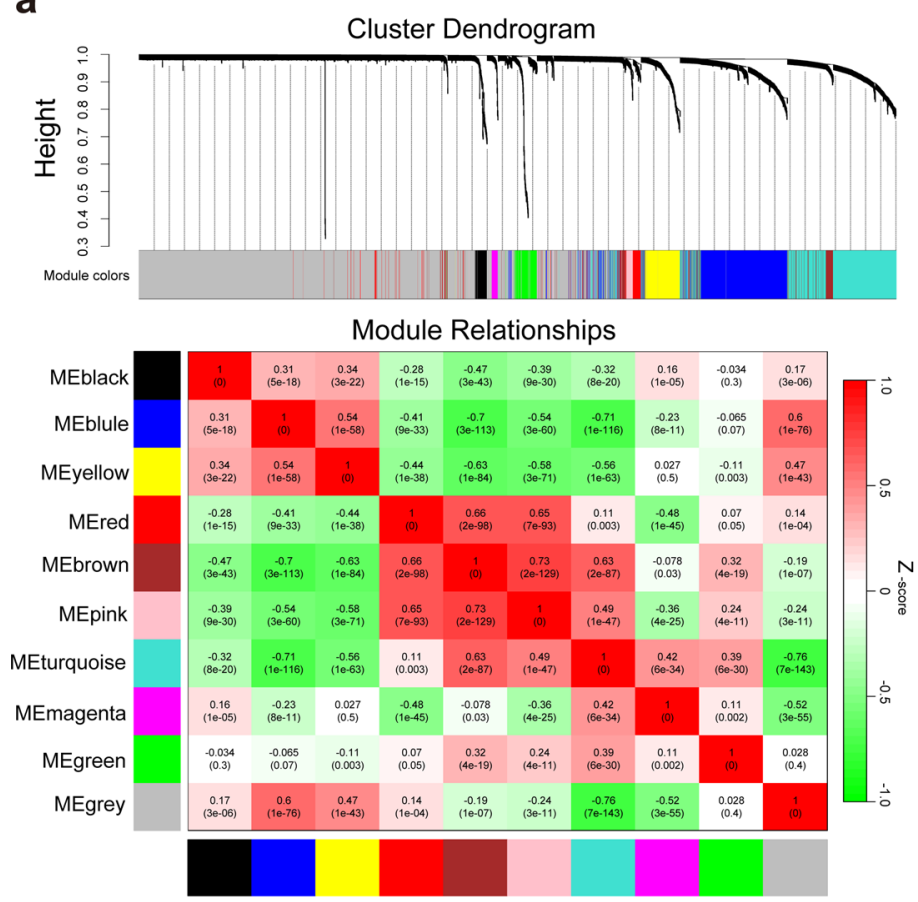

C

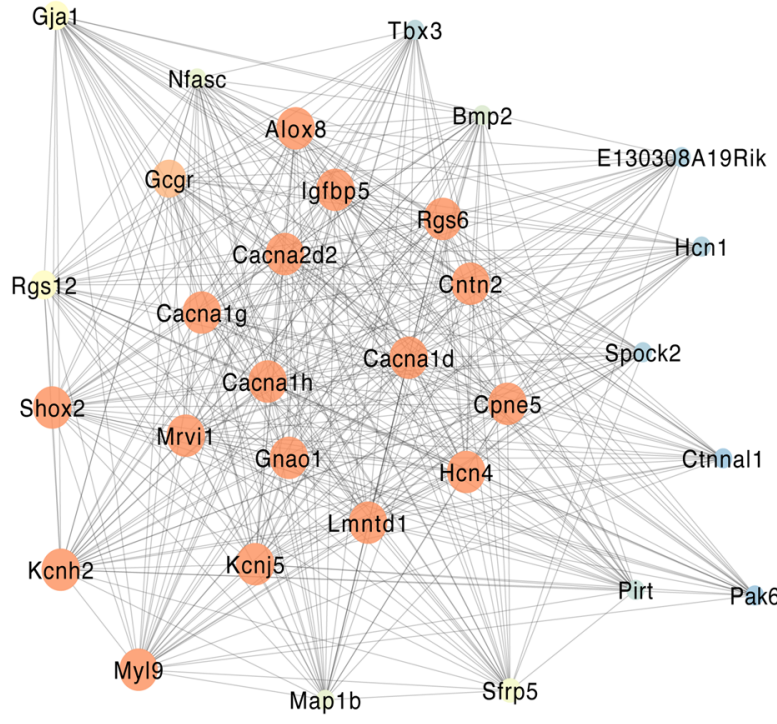

b

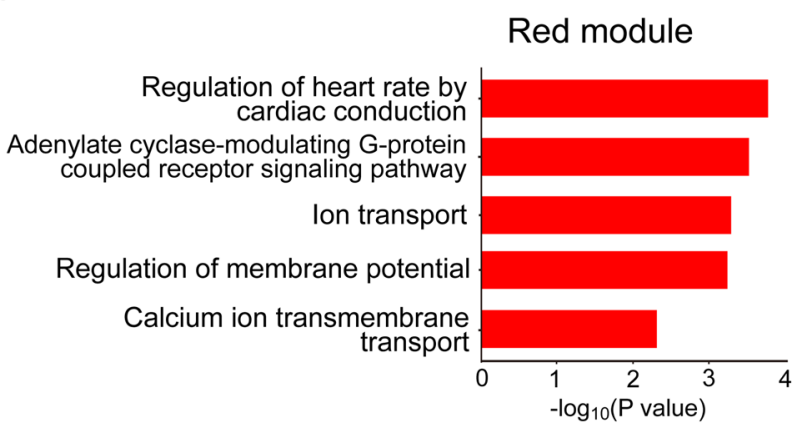

Brown module

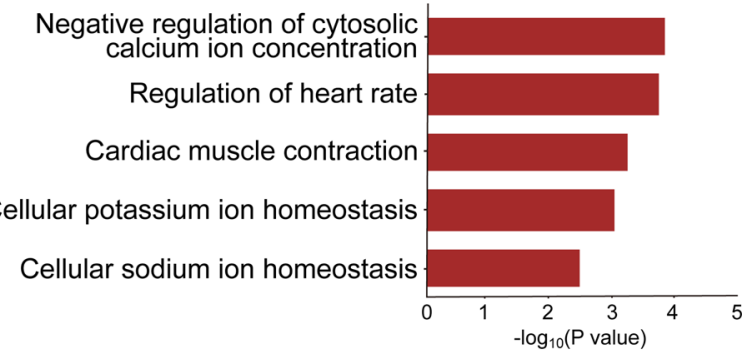

d

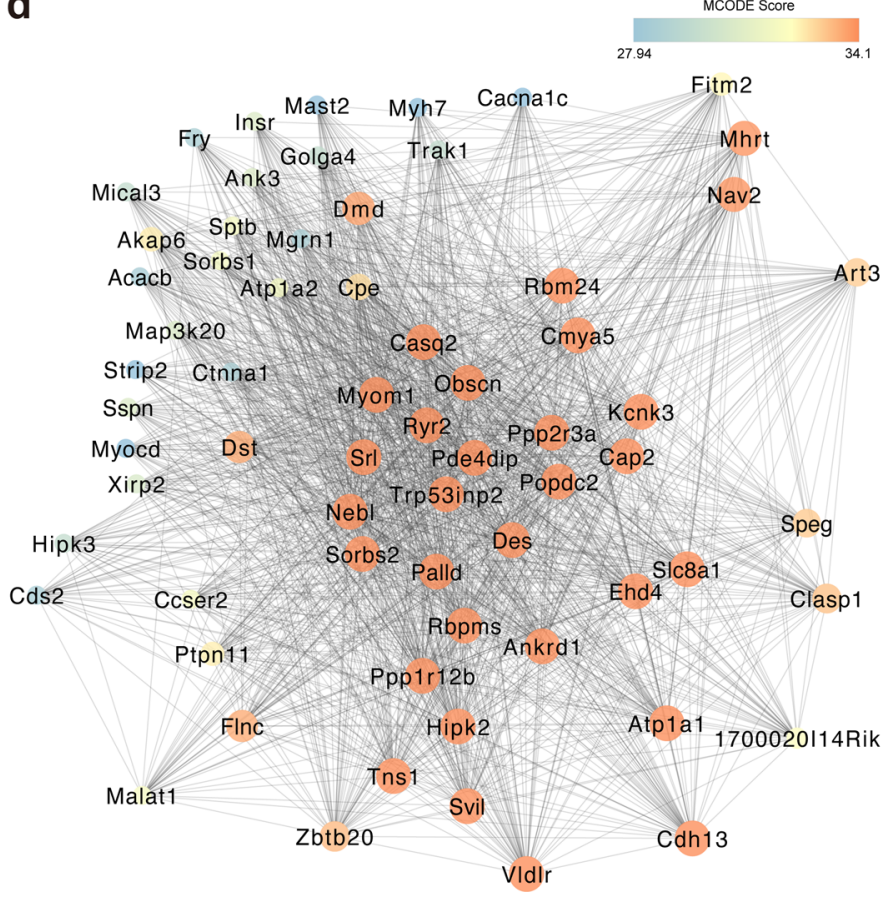

Fig. 5 Weighted gene co-expression network analysis (WGCNA) analysis of mouse sinoatrial node (SAN) cells. a The hierarchical cluster dendrogram identifies ten gene co-expression modules. Heat map shows the correlation of these modules. b Gene Ontology (GO) analysis shows that the main terms of red and brown modules were all related to SAN pacemaker activity. c Co-expression network of red module genes mainly focus on $\mathrm{K}^{+}$and $\mathrm{Ca}^{2+}$ channels. d Co-expression network of brown module genes mainly focus on $\mathrm{Ca}^{2+}$ regulators. The networks were created by $\mathrm{Cytoscape}$ and the high MCODE score of genes was mapped to the bright color of nodes.

protein-coupled receptor genes (ARRB1, RND1 and RGS5), and cell adhesion genes (F11R and ESAM) (Fig. 7b-d and Supplementary Data 6). It should be noted that numerous functional genes related to SAN function were not enriched in the DEGs of primate SAN clusters compared with mouse and rabbit, which may be due to the limited annotation of cynomolgus monkey genome. Many SAN-related genes were merely predicted, thus were not included in clustering analysis.
The key pacemaker activity genes expression analysis across species. Finally, to determine the species specificity of key gene expression patterns in different mammals, we further compared the expression of several functional genes associated with pacemaker activity common to mice, rabbits and cynomolgus monkey. We found that genes encoding $\mathrm{HCN}$ channels, $\mathrm{K}^{+}$channels, $\mathrm{Ca}^{2+}$ channels, transporters and $\mathrm{Ca}^{2+}$ regulators are all expressed in SAN of the three species assayed (Fig. 8). In contrast, the 
a

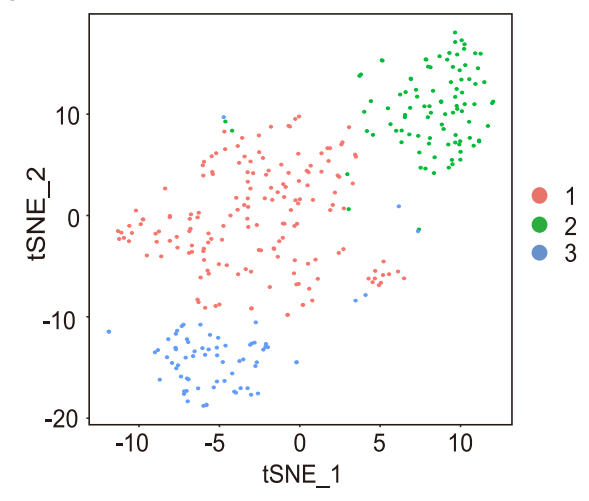

e

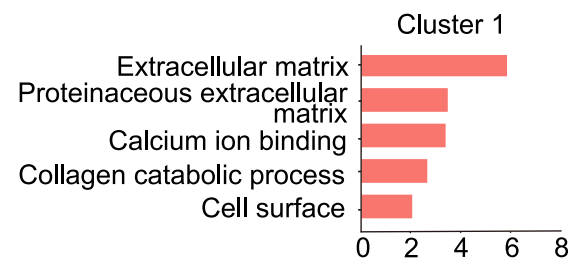

Cluster 2
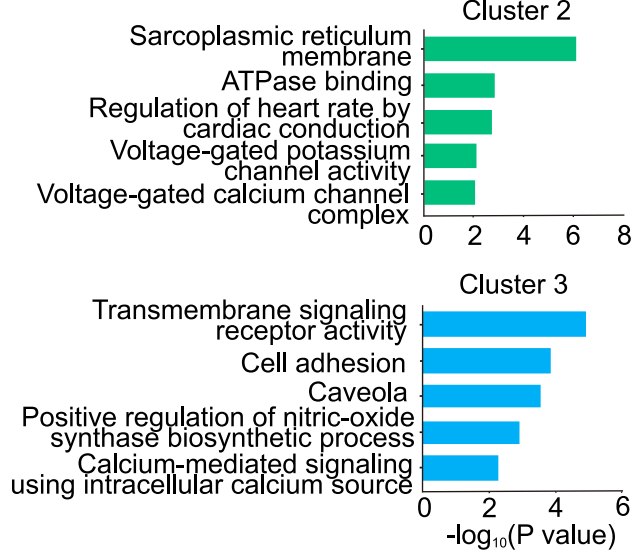

b

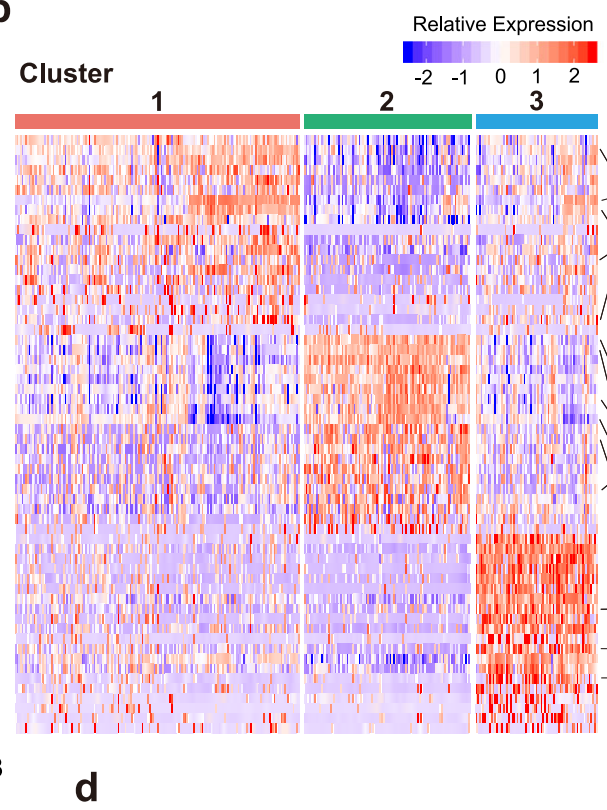

C
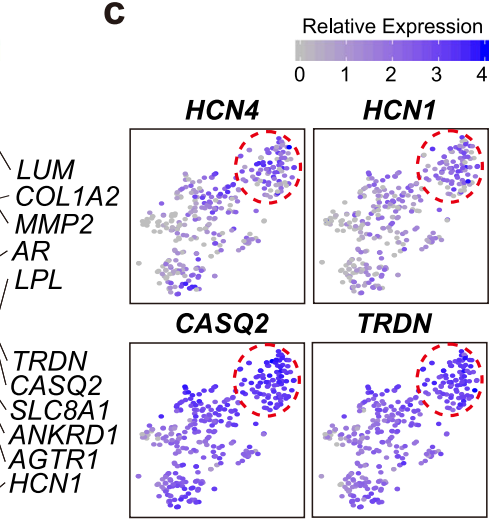

AGTR1

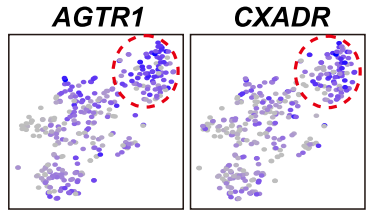

S1PR1

CD1D

SELE

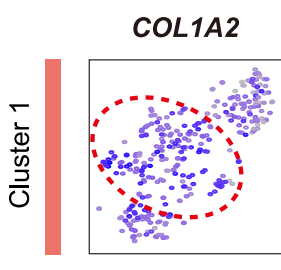

AR
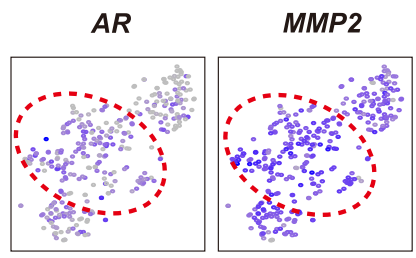

SELE
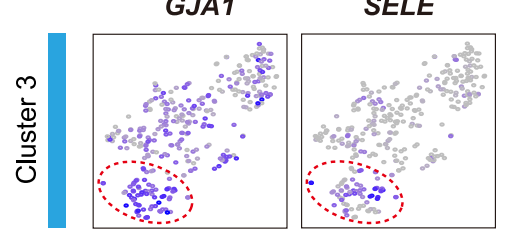

CD1D

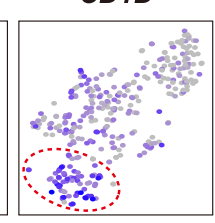

Fig. 6 Cell classification of rabbit sinoatrial node (SAN) cells. a t-distributed stochastic neighbor embedding (t-SNE) analysis identifies three-cell clusters in rabbit SAN ( $n=343$ biologically independent cells of six independent animals). b Heat map shows differentially expressed genes (DEGs) of each cluster. c Feature plot of specific DEGs in Cluster 2. d Feature plot of specific DEGs in Cluster 1 and Cluster 3. Location of different clusters was marked with the red circles. e Gene Ontology (GO) analysis of three clusters. Cluster 2 showed it is highly associated with regulation of heart rate, while Cluster 1 was enriched in extracellular matrix and Cluster 3 was related to cell adhesion besides ion channels regulation.

expression of Kcnd2 (encoding a potassium voltage-gated channel Kv4.2) was low in SAN cells of three species (Fig. 8b). Interestingly, Kcnj8, encoding the inwardly rectifying $\mathrm{K}_{\mathrm{ATP}}$ channel subunit Kir6.1, which has been reported to be present in mouse SAN pacemaker cells and involved in the regulation of heart rate $^{32}$, was not expressed in the potential core cell cluster of mouse and rabbit SAN (Fig. 8b).

\section{Discussion}

The field of SAN research has long been dominated by two major questions. First, are there different subsets of pacemaker cells in SAN? Second, what is the core molecular regulatory network of pacemaker activity in SAN? In this study, we preliminarily answered these two questions by transcriptionally profiling the SAN cells using scRNA-seq. On the one hand, we found that the SAN cells in mice can be divided into four clusters, including one core cell cluster, whereas SAN cells in rabbit and cynomolgus monkey can be divided into three clusters, each of which also contains a core cell cluster. On the other hand, we identified the core gene regulation network of pacemaker activity, which was consistent with the protein interaction analysis. The genes regulatory network underlying the pacemaker activity may govern the spontaneous depolarization and intrinsic automaticity of SAN pacemaker cells.

One of the bottlenecks in SAN pacemaker activity research is lack of specific cell-type markers. Hcn4 and Hcn1, the classical molecular markers of SAN ${ }^{33,34}$, are highly expressed in the SAN and cardiac pacemaker regions of different species and provides great convenience for the study of pacemaker cell biology and electrophysiology. The DEGs analysis in our study revealed three genes, including Vsnl1, Dlgap1, and Unc80, could be the possible candidate markers of core cell cluster in mouse SAN. However, the expression of Dlgap1 and Unc80 was less specific and relatively low abundance in SAN. We found that Vsnll was highly and specifically expressed in SAN compared to atrium and ventricle, functionally, it participates in cardiac cell rhythm regulation. Moreover, VSNL1 was also highly and specifically expressed in the rabbit SAN, and was one of the TOP DEGs in the core cell cluster of the monkey, suggesting the conservation of its abundant expression in SAN across species. All these make Vsnl1 a possible SAN marker. In addition, markers of AV cells not only define AV cells themselves but play an important complementary 
a

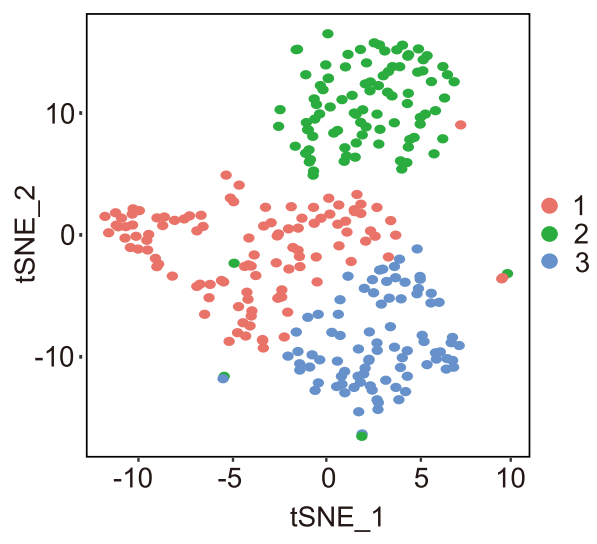

C
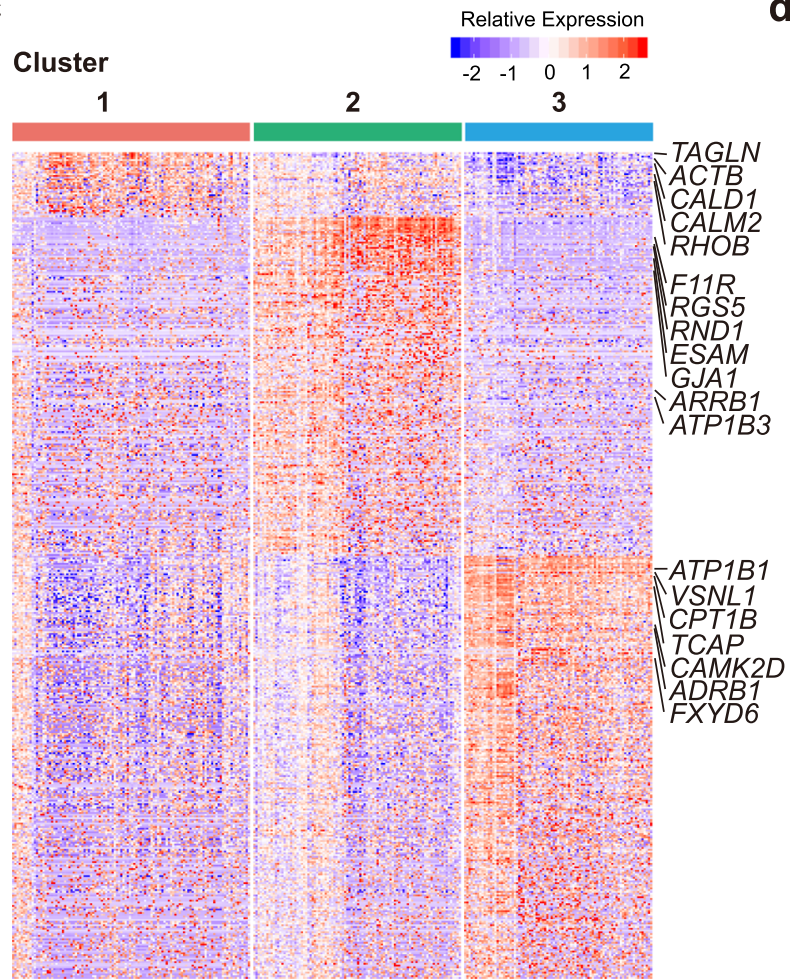

b
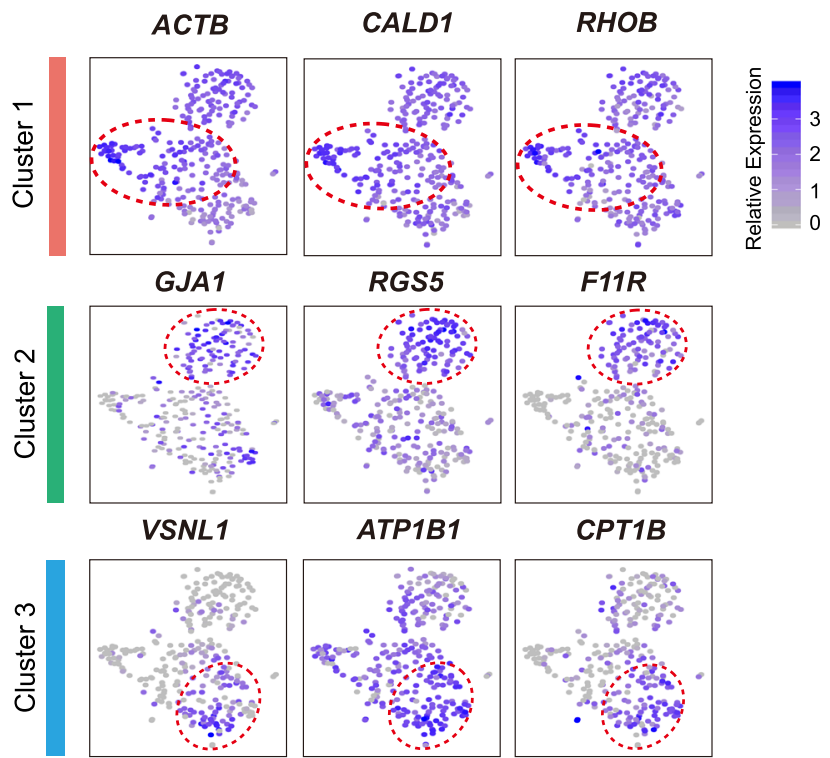

d

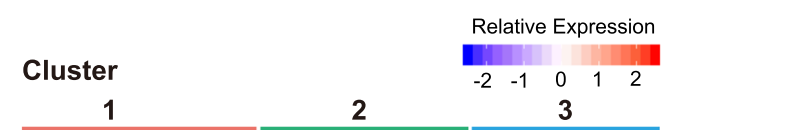

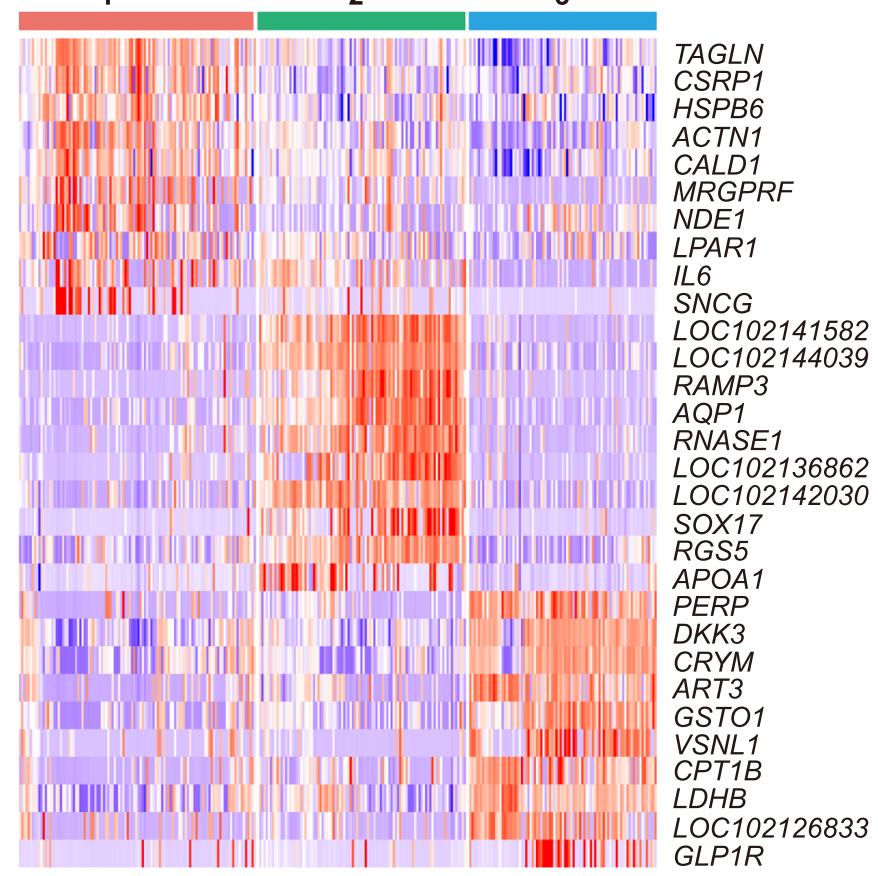

Fig. 7 Clustering analysis of cynomolgus monkey sinoatrial node (SAN) cells. a Three clusters of monkey SAN cells were identified by $t$-distributed stochastic neighbor embedding ( $t$-SNE) ( $n=296$ biologically independent cells of 12 independent animals). b Feature plot of specific differentially expressed genes (DEGs) in each cluster and Cluster 3 was identified as the core cell cluster. VSNL1 was also specifically expressed in Cluster 3. Location of different clusters was marked with the red circle. $\mathbf{c}$ Heat map shows all DEGs of each cluster. The main DEGs of each cluster were indicated in the right column. d Heat map shows top10 specific DEGs of each cluster.

role in distinguishing SAN cells from AV cells. Our study showed that Lrtm1, Ptgds, and Slc25a13 were highly expressed in AV cells and rarely expressed in the SAN cells. When combining the AV specific genes with SAN specific expression of Vsnll, it will be benefit to the identification of the SAN cells. Furthermore, the specific nature of the markers identified here may lead to lineage tracing for the development of SAN cells.

The Vsnll gene encodes visinin-like 1 protein, which belongs to the visinin-like protein subfamily of neuronal calcium sensor proteins. VSNL1 has been found to be involved in calcium signaling pathways and implicated in synaptic pathology in Alzheimer's disease ${ }^{35}$. A recent study analyzed the transcriptomes of late fetal FACS-purified Tbx $3^{+}$SAN nodal cells and NppbKatushka $^{+}$AV chamber cardiomyocytes, and identified a series of SAN-enriched gene program including Vsnll, which was also found enriched in fetal human SAN regions ${ }^{36}$, suggesting its potential role in SAN development. However, its function in SAN remains unclear. Our study revealed the effect of Vsnl1 in SAN through functional studies and gene expression analysis. We speculated that the Vsnl1 ${ }^{+}$core cells may have more important 
a

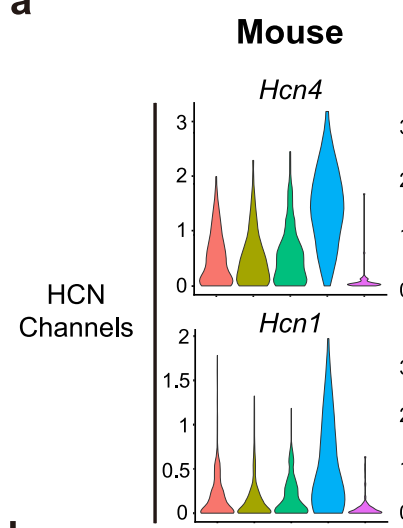

b

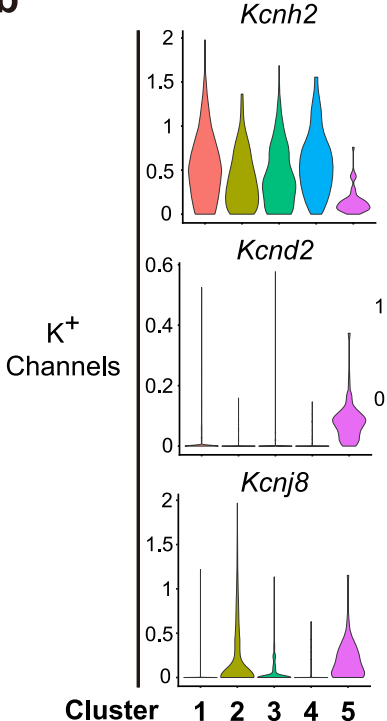

Rabbit

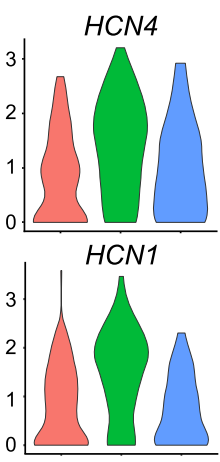

KCNH2
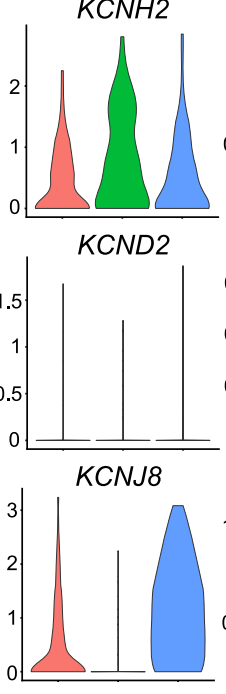

123
Monkey

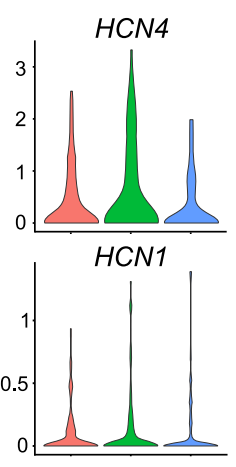

KCNH2
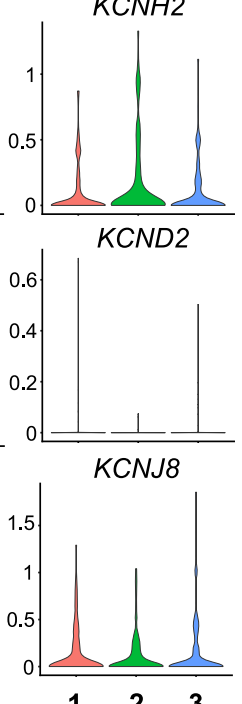

C

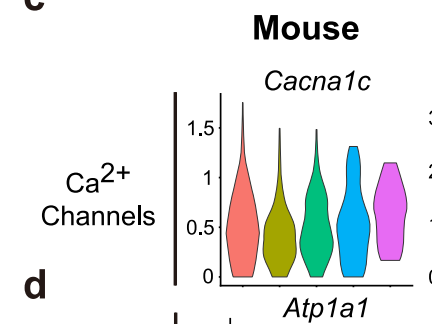

Rabbit

Monkey

CACNA1C CACNA1C
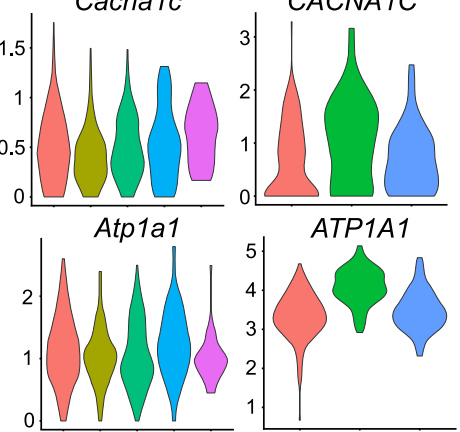

Transporters

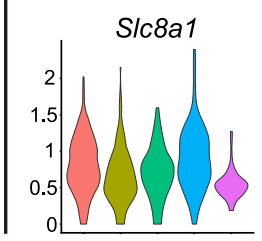

e
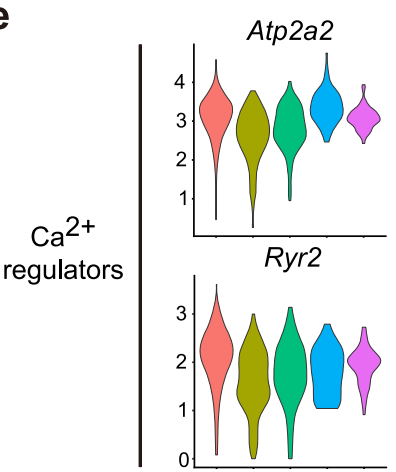

Ryr2
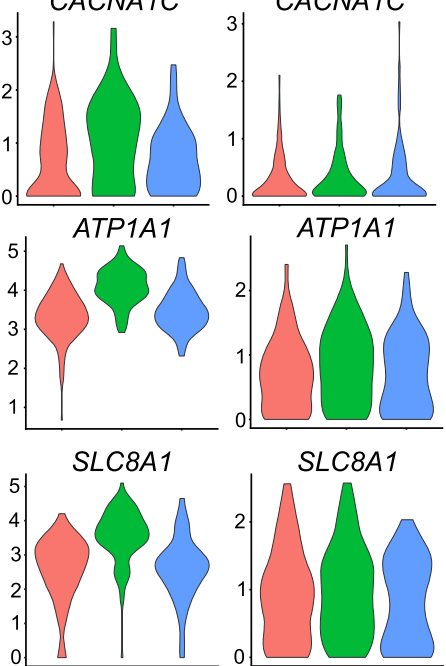

SLC8A1

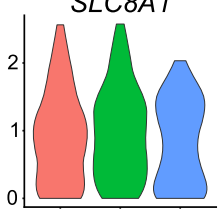

ATP2A2
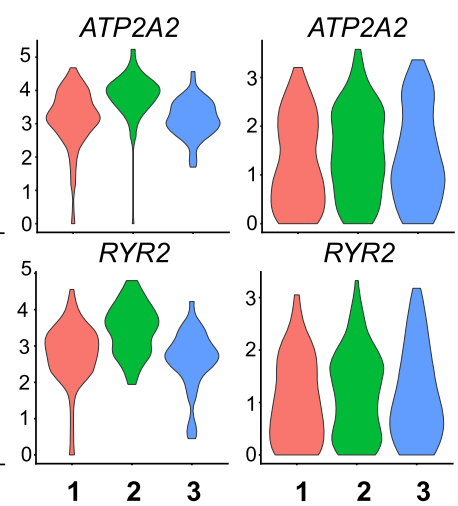

Fig. 8 Relative expression of key functional gene related to autonomic rhythm of sinoatrial node (SAN) cells among three species. a Expression of HCN channel genes. $\mathbf{b}$ Expression of $\mathrm{K}^{+}$channel genes. Kcnd2 was detected in atrial and ventricular (AV) cells but low expression in SAN cells. c Expression of $\mathrm{Ca}^{2+}$ channel genes. d, e Expression of transporter and $\mathrm{Ca}^{2+}$ regulator genes.

roles in SAN function compared to other clusters. Moreover, considering that the Vsnl1 ${ }^{+}$core cells are potentially diffusely distributed in SAN tissue, they may be the cellular basis of the "leading pacemaker" site and "pacemaker shift" phenomenon based on their non-restricted distribution within SAN. The specific expression of Vsnll in the SAN of different species may shed light on its research in human. The role of Vsnll in SAN function needs to be fully established in the future study. Our findings also open avenues in the investigation of lineage tracing of pacemaker cells, cardiac autonomic rhythm and biological pacemaker.

The hiPSC-CM model was used to investigate the effect of cell cluster marker genes on frequency in vitro. However, the hiPSC$\mathrm{CM}$ is primarily used as an approach to study cellular phenotypes of inheritable cardiac diseases and provide in vitro platforms for mechanistic investigation in regenerative medicine ${ }^{37-39}$. In the field of cardiac electrophysiology, the hiPSC-CM could be used as a cellular model for some pacemaker mechanism studies because there are some similarities between hiPSC-CMs and pacemaker cells such as the presence of spontaneous firing rate and the reactivity to isoproterenol and nifedipine ${ }^{40,41}$. However, hiPSCCMs cannot be justified as surrogate for SAN pacemaker cells. hiPSC-CMs compose mixed cardiac cell types showing immature phenotypes, small action potentials, and fetal-like transcriptome profiles; importantly, they also exhibit heterogeneous electrophysiology phenotypes and spontaneous automaticity ${ }^{42}$. Furthermore, the regulation mechanisms underlying the pacing of
SAN cells and hiPSC-CMs are different as well. The pacing of SAN cells is thought to be initiated and maintained by a membrane clock and a $\mathrm{Ca}^{2+}$ clock, whereas the initiation and frequency of spontaneous pacing in hiPSC-CMs is thought to be mainly depends on localized spontaneous $\mathrm{Ca}^{2+}$ releases via RYR and other $\mathrm{Ca}^{2+}$ regulators such as SERCA2 and NCX, which could be independent of membrane depolarizations generated by ion channels ${ }^{43-45}$.

The gene expression analysis after Vsnl1 knockdown suggests the different mechanisms of Vsnll in frequency regulation in different cell types. The pluripotent stem cells derived SAN pacemaker-like cell would be a more ideal model for exploring the ionic basis of SAN function in vitro. However, most cardiac differentiation models based on pluripotent stem cells are used to simulate ventricular cardiomyocytes, whereas approaches for the generation of SAN-like pacemaker cells are sparse with low efficiency 39,46 . The development of sophisticated differentiation protocol to efficiently produce pacemaker-like cells will definitely bring an opportunity to the study of SAN.

The intrinsic rhythm of SAN is regulated by the autonomic nervous system ${ }^{47,48}$ as well, although in ways still poorly understood to date. In this study, autonomic nerve signaling/G protein-coupled receptor genes were identified as important elements of pacemaker activity in core gene regulatory network. Notably, KCNJ5, encoding an inwardly rectifying potassium channel Kir3.4, was included in the core gene regulatory network. 
Kir3.4 is responsible for the acetylcholine/adenosine-induced potassium $I_{\text {KACh }}{ }^{49}$. The vagal release of acetylcholine stimulates muscarinergic activation of an inhibitory $G$ protein, which in turn opens the Kir3.4 channel, the SAN pacemaker cells are then repolarized or hyperpolarized by activating $I_{K A C h}{ }^{50}$. Interestingly, a specific glucagon receptor Gcgr, which has been linked to the regulation of heart rate ${ }^{51,52}$, was also present in core gene regulatory network and may be involved in the regulation of $G$ protein-coupled receptor signaling in SAN, suggesting the nonion channel genes may critically affect SAN function as well. The results of WGCNA could also provide directional and systematic strategy for mechanistic investigation.

One of the exciting aspects of current SAN research is the relationship between transcription factors and SAN development $^{53}$. In this study, it was found that two transcription factor genes, i.e., Shox 2 and $T b \times 3$, which are essential for the formation and function of cardiac conduction system ${ }^{53}$, are members of the core gene regulatory network of adult SAN cells. These transcription factors interact with and co-regulate each other. For example, Shox2 expression is crucial for SAN development and is restricted to the sinus venosus, where it prevents the formation of working myocardium by inhibiting $N k \times 2-5$ expression and activating Hcn4, Isl1, and Tbx3 expression ${ }^{54,55}$. Our WGCNA data revealed that these transcription factors and the relevant regulatory network may also play an important role in SAN pacemaker activity.

The basic proteins involved in SAN pacemaker activity mainly include ion channels, transporters, $\mathrm{Ca}^{2+}$ regulators, autonomic nerve signaling/G protein-coupled receptors, and transcription factors $^{3,10,55}$. Our work shows that these types of components all exist to some extent in the core gene regulation network governing the function of SAN cells in different species. Therefore, the classical theoretical framework of pacemaker cell autonomic rhythm may have potential species universality. However, it must be pointed out that, in terms of specific hub regulatory molecules, there is a degree of difference among the three species analyzed in this study. For instance, in rabbit, high expression of transporter genes and $\mathrm{Ca}^{2+}$ regulator genes are more obvious in core cell clusters (Fig. 8d, e). Thus, caution should be exercised in analyzing the mechanisms underlying SAN pacemaker activity across species.

In summary, our work reveals the molecular and cellular maps of SAN cells at single-cell resolution, providing systematic data for the origin and regulation of SAN pacemaker activity research. These findings are of fundamental significance in the in-depth understanding of biology and pathogenesis of SAN, as well as its interventions ${ }^{56}$

\section{Methods}

Animals. This study conformed to the rules of the Guide for the Care and Use of Laboratory Animals made by the U.S. National Institutes of Health. All of the animal experiments were approved by the Animal Care and Use Committee of Tongji University School of Medicine (mouse and rabbit experiments), or Institutional Animal Care and Use Committee of Suzhou Xishan Zhongke Drugs Research and Development Centre (monkey experiments). Breedings of mice were kept under SPF conditions in a $12 \mathrm{~h}$ dark-light-cycle environment with food and water ad libitum. Ambient temperature was $22^{\circ} \mathrm{C}$ and humidity $60 \%$.

Isolation of single cells. Single SAN cells were isolated from male adult mice, New Zealand White rabbits, and cynomolgus monkeys, respectively, based on previously described methods ${ }^{13,57,58}$. The heart was removed quickly and placed in the prewarmed Tyrode's solution containing: $140 \mathrm{mM} \mathrm{NaCl}, 5.4 \mathrm{mM} \mathrm{KCl}, 1.2 \mathrm{mM}$ $\mathrm{KH}_{2} \mathrm{PO}_{4}, 1.8 \mathrm{mM} \mathrm{CaCl}_{2}, 1.0 \mathrm{mM} \mathrm{MgCl}, 5.5 \mathrm{mM}$ glucose, and $5 \mathrm{mM}$ HEPES (pH was adjusted to 7.4 with $\mathrm{NaOH}$ ). The solution was continuously oxygenated with $\mathrm{O}_{2}$. The SAN was dissected from the region bordered by the crista terminalis, the superior and inferior vena cavae. The SAN tissue was then cut into small pieces and washed twice in low-Ca ${ }^{2+}$ solution containing: $140 \mathrm{mM} \mathrm{NaCl}, 5.4 \mathrm{mM} \mathrm{KCl}$, $1.2 \mathrm{mM} \mathrm{KH}_{2} \mathrm{PO}_{4}, 0.2 \mathrm{mM} \mathrm{CaCl}_{2}, 18.5 \mathrm{mM}$ glucose, $50 \mathrm{mM}$ taurine, and $1.0 \% \mathrm{BSA}$ ( $\mathrm{pH}$ was adjusted to 6.9 with $\mathrm{NaOH}$ ). The SAN was then digested at $36-37^{\circ} \mathrm{C}$ for
20-25 min in the low- $\mathrm{Ca}^{2+}$ solution containing elastase $(0.3 \mathrm{mg} / \mathrm{ml}$; Worthington NJ, USA), collagenase type $2(0.8 \mathrm{mg} / \mathrm{ml}$; Worthington, NJ, USA), and protease $(0.13 \mathrm{mg} / \mathrm{ml}$; Sigma, Chemical Co.) with gentle agitation. The concentration of the enzyme was adjusted to achieve an optimized digestion in different species. The digestion was stopped by transferring the pieces to the modified Kraftbruhe (KB) solution, containing $100 \mathrm{mM}$ potassium glutamate, $10 \mathrm{mM}$ potassium aspartate, $25 \mathrm{mM} \mathrm{KCl}, 10 \mathrm{mM} \mathrm{KH}_{2} \mathrm{PO}_{4}, 2 \mathrm{mM} \mathrm{MgSO}_{4}, 20 \mathrm{mM}$ taurine, $5 \mathrm{mM}$ creatine, $0.5 \mathrm{mM}$ EGTA, $20 \mathrm{mM}$ glucose, $5 \mathrm{mM}$ HEPES, and 1.0\% BSA (pH was adjusted to 7.2 with $\mathrm{KOH}$ ). The SAN pieces were then dissociated by pipetting gently in the KB solution with a fire-polished glass pipette.

The AV myocytes were isolated as previously described ${ }^{59}$. The heart was removed and perfused with a low- $\mathrm{Ca}^{2+}$ solution containing $117 \mathrm{mM} \mathrm{NaCl}, 4 \mathrm{mM}$ $\mathrm{KCl}, 10 \mathrm{mM}$ HEPES, $1 \mathrm{mM} \mathrm{KH} \mathrm{PO}_{4}, 4 \mathrm{mM} \mathrm{NaHCO}, 1.7 \mathrm{mM} \mathrm{MgCl}_{2}, 0.1 \mathrm{mM}$ $\mathrm{CaCl}_{2}$, and $10 \mathrm{mM}$ glucose. Then, the heart was perfused with low-Ca ${ }^{2+}$ solution containing $300 \mathrm{U} / \mathrm{ml}$ collagenase type 2 (Worthington, NJ, USA) for 15-20 min. When the heart became slightly pale and flaccid, the atrium and ventricle were removed and cut into small pieces in the $\mathrm{KB}$ solution, respectively. The tissue was dissociated through pipetting and then filtered through a cell strainer $(100 \mu \mathrm{m} ; \mathrm{BD}$ Falcon, NJ, USA).

Single-cell RNA sequencing. Single-cell RNA sequencing was performed using Smart-seq 2 protocol with minor modification ${ }^{60,61}$. In brief, single cell was pipetted into $0.5 \mu \mathrm{l}$ lysis buffer under the microscope to release the total RNA. RNA was reversed to first-strand cDNA through nine cycles using Superscript III (Invitrogen, 18080044) and then was amplificated for 18 PCR cycles using KAPA polymerase (Kapa Biosystems, KK2601). The RT-PCR product was purified using Agencourt AMPure XP Beads (Beckman Coulter, A63881), and then 0.1 ng cDNA was used for Nextera tagmentation (Illumina, FC-131-1096) to construct library. The ERCC spike-in (Invitrogen, 4456740) was added in reverse transcription mix during the library construction according to the Smart-seq 2 protocol. Finally, the libraries were sequenced with paired end $2 \times 150$ bp reads on Illumina HiSeq X10.

Single-cell RNA sequencing data processing. Raw sequencing reads from singlecell RNA sequencing libraries were trimmed to remove adapter sequences, which removed the leading and trailing low-quality bases below quality 3 or $\mathrm{N}$ bases, cut the sliding window which average quality per base drops below 15, and dropped reads below the 36 bases long (Version Trimmomatic- 0.33 ). The clean reads were aligned to the mouse genome (GRCm38 assembled by UCSC), the rabbit genome (oryCun2 assembled by UCSC), or cynomolgus monkey genome (macFas5 assembled by UCSC) with Hisat2 (version 2.0.5). The mapping rate was $32.32 \%$ when measured using the raw reads to align to mouse reference genome sequence, while the mapping rate is $42.35 \%$ when mapped to the mouse reference genome using the trimmed reads. The gene annotation file for mouse was downloaded from the database GENCODE (release 20) ${ }^{62}$. Gene annotation files of cynomolgus monkey and rabbit obtained from UCSC were organized into the RefSeq Genes tracks using the table tool, respectively. Unique mapped reads were counted with HTSeq (version 0.9 .1$)^{63}$ and grouped according to the sample name. For a given cell, the number of reads was counted up within a gene. This read count quantitation was used to estimate the gene expression of each gene in the cells and the read count matrix was used for the subsequent analysis. Single cells with more than 200 genes detected were used for downstream analysis.

The key functional genes described in Fig. 8 are predicted genes, which are not annotated in RefSeq Genes tracks in cynomolgus monkeys. We extracted these genes using ENSEMBL database and aligned them to macFas5 to calculate the read count. For mouse, the relative expression of functional genes was calculated in the clusters separately.

Identification of cell clusters and DEGs. Raw counts were used as the input of Seurat R package (version 2.3). In the process, count data were normalized and logtransformed by "NormalizeData" function, which normalized the read counts for each cell by the total gene expression through multiplies this value by a scale factor $(10,000)$ and log-transforms the result. Then the normalized read counts were used in the downstream analysis. Overall, using "FindVariableGenes" function, 7733 highly variable genes were obtained to perform principal component analysis (PCA). After that, significant PCs were identified through the "JackStraw" function with default parameters. Fourteen PCs were selected as the significant components for $\mathrm{t}$-SNE analysis. We then set the clustering parameter resolution to 0.6 for the "FindClusters" function in Seurat to identify cell clusters. Among the clusters, DEGs were identified using the function "FindMarkers" and "FindAllMarkers" of Seurat Package with min.pct set to 0.25 . Top DEGs were presented according to the $\log$ fold-change of the average expression (avg.logFC) and adjusted $p$ value (p.val. adj.). Finally, GO enrichment analysis was performed using DAVID (version 6.8).

For mouse, we screened out 2500 genes which are enriched in mouse heart basing on previously report and performed the cell cluster analysis ${ }^{6}$. The expression data of these genes were extracted and processed as described above.

Weighted gene co-expression analysis. The read count data were pre-processed before WGCNA analysis (version 1.64). First, the read counts with less than 10 were filtered out to remove low-expressed features and then 3831 genes left. Then, 
the read counts of these filtered 3831 genes were normalized with the function "Variance Stablizing Transformation" (VST) in DESeq2 package (version 1.22.2).

The normalized data were clustered to examine possible obvious outliers. Then we use the "pickSoftThreshold" function to choose a proper soft-thresholding power for network construction. The power 4 was found to be the lowest power for which the scale-free topology fit index curve flattens out upon reaching a high value. Based on this power, a signed weighted correlation network was constructed and modules were identified. The parameter mergeCutHeight (0.25) was used for merging of modules.

Module eigengenes were used to measure the module membership (also known as module eigengene based connectivity kME). Genes with highest module membership values were referred as intramodular hub genes $(\mathrm{kME}>0.8)$. Intramodular hub genes were centrally located inside the module and represented the expression profiles of the entire module.

Cytoscape (version 3.6.1) was used to visualize the gene connections in different modules. Using the MCODE (version 1.5.1) to find the network of a cluster, which contained 31 nodes and 417 edges in whole mouse red module genes (Score: 27.8), and this network was laid out using "Prefuse Force Directed Layout" according to the weight. The visualization of the network was set to the high MCODE score of gene nodes with the large size and the bright color. The mouse brown module and rabbit turquoise module were analyzed using the same way. The cluster (Score: 48.123) contained 66 nodes and 1564 edges was found in the mouse brown module. The cluster in rabbit turquoise module (Score: 52.933) contained 61 nodes and 1588 edges.

STRING (version 11.0) was used for prediction and integrating the protein-protein interaction coding by module genes. Genes were inputted into the "Multiple Proteins" term and meaning of network edges was set to confidence. Hub proteins with its connected nodes were displayed.

Quantitative real-time PCR. Total RNA of adult mouse SAN and atrium tissues was isolated using GenElute Single Cell RNA Purification Kit (Sigma, RNB300), total RNA of adult mouse ventricle, adult rabbit heart tissues, and neonatal rat cardiomyocytes were extracted using Trizol reagent (Thermo Fisher Scientific). Purified RNA was reverse-transcribed by the PrimeScript RT Reagent kit (Takara Bio, RR037A). Quantitative real-time PCR (qPCR) was performed using SYBR Green kit (TOYOBO CO, QPK-201) on the QuantStudio ${ }^{\text {TM }} 6$ Flex system (Applied Biosystems; Thermo Fisher Scientific). GAPDH was used for endogenous control and the relative expression of mRNA level was quantified using $2^{-} \triangle \triangle \mathrm{CT}$ method. The primers sequences are listed in Supplementary Data 7.

Histology and immunofluorescence histochemistry. After anesthesia, the hearts of adult mice (8-12-week-old mice) were quickly removed, then atrium and peripheral connections were preserved. After rinsing with cold PBS, the hearts were fixed with $4 \%$ paraformaldehyde (PFA; Sigma) overnight at $4{ }^{\circ} \mathrm{C}$. Next, hearts were dehydrated in increasing concentrations of ethanol and embedded in paraffin. Hearts were sectioned in $6 \mu \mathrm{m}$ in horizontal section. The SAN of adult mice is located in the posterior wall of the right atrium, in the intercaval region adjacent to the atrial muscle of the crista terminalis, extending from the superior to near the inferior vena cava (Supplementary Fig. 1a). The SAN is surrounded by connective tissue, and the SAN node artery passing through the area. SAN area was labeled by staining $\mathrm{HCN} 4$, atrium was labeled by Connexin $43, \mathrm{HCN} 4$ was abundantly present throughout the entire SAN area, while Connexin 43 was detected exclusively in the atrium (Supplementary Fig. 1b).

After deparaffinization, re-hydration, and microwaving antigen retrieval in citrate solution, paraffin-embedded heart slices were blocked with $10 \%$ goat serum (Invitrogen) and incubated with primary antibody overnight at $4^{\circ} \mathrm{C}$. Next day, the slices were washed in PBST for three times and then incubated with respective fluorescent secondary antibody (Invitrogen) for $1 \mathrm{~h}$ at room temperature. The slices were washed again in PBST for three times then stained with DAPI (Sigma) to label the nuclei. Primary antibodies included HCN4 (Sigma, SAB5200035, 1:50), Connexin 43 (CST, 3512, 1:50), VSNL1 (Gene Tex, GTX115039, 1:50), Collagen I (Abcam, ab21286, 1:50), DLGAP1 (Affbiotech, AF0308, 1:50), UNC80 (BIOSS, BS12121R, 1:50), APOLD1 (Novus Biologicals, NBP2-58460, 1:50), RYR3 (Novus Biologicals, NBP2-76962, 1:50), Connexin 40 (Invitrogen, 37-8900, 1:50), cTNT (Abcam, ab8295, 1:50). Secondary antibodies included Goat anti-Mouse IgG Alexa Fluor $^{\circledR} 488$ (Abcam, ab150113, 1:200), Goat anti-Rabbit IgG Alexa Fluor ${ }^{\circledR} 555$ (Abcam, ab150078, 1:200), and Goat anti-Mouse IgG Alexa Fluor ${ }^{\oplus} 633$ (Sigma, SAB4600139, 1:200). Pictures were taken from a confocal microscope (Leica TCS SP8).

Culture and transfection of hiPSC-derived CMs. hiPSC-derived CMs were purchased from Help Stem Cell Innovations (Nanjing, China). hiPSC- derived CMs were routinely maintained in cardiomyocytes culture medium (Help Stem Cell Innovations, HELP4001) and medium was changed every $48 \mathrm{~h}$. After 18 days, hiPSC-derived CMs were dissociated with cardiomyocytes dissociation medium (Help Stem Cell Innovations, HELP4005) for $5 \mathrm{~min}$ at $37^{\circ} \mathrm{C}$, and then seeded onto MEA plates $\left(5 \times 10^{5}\right.$ cell $/$ plate). Cultures were maintained at $37^{\circ} \mathrm{C}$ with $5 \% \mathrm{CO}_{2}$. Transfection of small interfering RNA (siRNAs) was done with the lipofectamine
RNAiMAX (Invitrogen, 13778-150), according to the manufacturer's guidelines. All siRNA sequences are listed in Supplementary Table 1.

MEA recordings. MEA assay was performed as previously described ${ }^{41,64}$. The hiPSC-derived CMs were seeded on a fibronectin-coated Maestro MEA 24-well plate at a density of 50,000 cells per well to obtain spontaneously beating monolayers. The hiPSC- derived CMs were then transfected with siRNA-targeting candidate genes (sequence provided in Supplementary Table 1) with RNAiMAX (Invitrogen, 13778-150). The electrical activity was recorded with Maestro system (Axion Biosystems Inc) at day 4 after transfection following a 15-min equilibration. The raw data were analyzed with Axion's platform software to measure beat period (s). Statistical analysis was performed with GraphPad.

Isolation and culture of neonatal rat cardiomyocytes. The neonatal rat cardiomyocytes were isolated from 1- to 3-day-old Sprague-Dawley rats. The ventricles were separated from hearts and cut into pieces $\left(\sim 1-3 \mathrm{~mm}^{2}\right)$. Then these pieces were dissociated in calcium-free HBSS (Gibco, 14175095) containing $0.125 \mathrm{mg} / \mathrm{ml}$ trypsin (Gibco, 15090046), $0.1 \mathrm{mg} / \mathrm{ml}$ collagenase type IV (Sigma, C4-22-1G), and $10 \mathrm{mg} / \mathrm{ml}$ DNase II (Sigma, D8764). Digestion was performed at $37^{\circ} \mathrm{C}$ and heart sections were continually stirred. The supernatant was then collected into HBSS containing 10\% FBS every $5 \mathrm{~min}$. The digestion procedure was repeated approximately 8-10 times until the tissues were completely digested. The supernatant was centrifuged at 1000 r.p.m. for 10 min and resuspended in DMEM (Gibco, 11965092) supplemented with 10\% FBS and $100 \mathrm{mM}$ 5-bromo-20-deoxyuridine (Sigma, B5002). The resuspended cells were passed through a cell strainer (100 $\mu \mathrm{m}, \mathrm{BD}$ Falcon), and then seeded onto $100-\mathrm{mm}$ plastic dishes for $2 \mathrm{~h}$ at $37^{\circ} \mathrm{C}$ to remove fibroblasts. The isolated neonatal rat cardiomyocytes were plated on $1 \%$ gelatin-coated plastic culture dishes at an appropriate density. After $24 \mathrm{~h}$, the medium was changed to low-serum medium, and the cells were cultured for $48 \mathrm{~h}$ then for use. The gene expression analysis after Vsnll knockdown was performed in neonatal rat cardiomyocytes transfected with siRNA (Vsnll) using Lipofectamine RNAiMAX (Invitrogen, 13778-150).

AAVs generation. The Vsnll-targeting miRs were designed using Invitrogen's online software (Invitrogen's RNAi Designer: http://rnaidesigner.thermofisher. com/rnaiexpress/design.do; Target Design Options: miR RNAi). According to the software, six miRNAi sequences targeting the different positions of Vsnll gene (Vsnl1-miRNAi) and negative control sequence (Control-miRNAi) were designed, synthesized, and cloned into the AAV2/9-CMV_bGI-eGFP-miRNAi plasmid vectors. The pAAV2-CMV bGI-eGFP-miRNAi (Vsnll) and pAAV2-CMV bGIeGFP-miRNAi (Control) plasmids were constructed using the BLOCK-iT Pol II miR RNAi Expression Vector Kits (Invitrogen, K493500) and the microRNA-based silencing technique (Taitool Bioscience) ${ }^{65}$. Then the vectors were co-transfected with the VSNL1-overexpressed plasmid into HEK293 cells, respectively, and the interference efficiency of each sequence is detected by western blot. Of the six miRNAi sequences, two validated miRNAi sequences (Target sequence 1: TGAGTTCAATGAGCATGAGCT; Target sequence 2: AGTGGAGATGCTGGA GATTAT) targeting the different positions of Vsnll gene were packaged into the virus vectors, respectively. The virus was then injected in adult male mice through tail vein, and their effectiveness was further confirmed in mouse SAN by qRT-PCR The most effective virus (Target sequence 1: TGAGTTCAATGAGCATGAGCT) was chosen and the corresponding miRNAi sequence as follows: $5^{\prime}$-TGCT GAGCTCATGCTCATTGAACTCAGTTTTGGCCACTGACTGACTGAGTTCA GAGCATGAGCT-3'

The telemetric recordings. For virus-injected mice, following vectors were used AAV2/9-CMV_bGI-eGFP-miRNAi (AAV9-miRNAi-Vsnll) (titer: $1.92 \times 10^{13}$ v.g. ml; Taitool Bioscience) and AAV2/9-CMV_bGI-eGFP-miRNAi (AAV9-miRNAicontrol) (titer: $1.70 \times 10^{13} \mathrm{v}$.g. $/ \mathrm{ml}$; Taitool Bioscience). All viral vectors were aliquotted and stored at $-80^{\circ} \mathrm{C}$ until use. Adult male mice were injected with a dose of $2 \times 10^{11}$ viral genome particles through tail vein. Fluorescence microscopy confirmed the virus infection in mouse SAN (Supplementary Fig. 15) and the Vsnl1 knockdown was confirmed in SAN regions (Supplementary Figs. 8 and 9). The mice were implanted with telemetric transmitters (ETA-F10; Data Sciences International) under $1-2 \%$ isoflurane anesthetization 1 week after virus injection. Telemetric transmitters were implanted into the peritoneal cavity of the mice and the paired wire electrodes were placed over the thorax, as previously described 66,67 Then the mice were housed in the single cage and exposed in $12 \mathrm{~h}$ dark/light cycle environment. Mice were given 2 weeks to recover from surgery before ECG recording. Recording and analysis parameters were set according to the manufacturer's instructions using Ponemah software (Datasci International)

Western blot. Adult rabbit SAN and AV tissue proteins were extracted by RIPA lysis buffer (Beyotime Biotechnology, P0013C) containing protease inhibitor cocktail (Roche, 04693132001$)$. Equal total proteins $(80 \mu \mathrm{g})$ were separated by $10 \%$ SDS-PAGE (Thermo Fisher Scientific, NP0315BOX), and then transferred onto PVDF membranes (Millipore, IPVH00010) The membranes were blocked with 5\% nonfat milk in TBST buffer for $1 \mathrm{~h}$ at room temperature and then incubated with VSNL1 antibody (Gene Tex, GTX115039, 1:1000) and GAPDH (Proteintech, 60004-1-Ig, 1:8000) overnight at $4^{\circ} \mathrm{C}$. On the next day, the membranes were 
incubated with conjugated secondary antibody (Invitrogen, A32730; Invitrogen, A32735) for $1 \mathrm{~h}$ at room temperature and the bands were visualized using the Odyssey imager.

Statistical analysis. Statistical analysis was performed with GraphPad Prism 8.0. The qPCR data were tested using Dunnett's multiple comparisons test and unpaired, two-tailed Student's $t$-test. The data are represented as the mean \pm s.e.m.

Reporting summary. Further information on research design is available in the Nature Research Reporting Summary linked to this article.

\section{Data availability}

The authors declare that the data supporting the findings of this study are available within the article and Supplementary Information files. The single-cell RNA-sequencing data have been deposited in the NCBI Sequence Read Archive (accession number of BioProject is PRJNA531288). All remaining data will be available from the corresponding author upon reasonable request. Source data are provided with this paper.

\section{Code availability}

The codes used in this study are available in Github [https://github.com/xue-lab/scRNAseq-of-sinoatrial-node].

Received: 8 May 2019; Accepted: 3 December 2020;

Published online: 12 January 2021

\section{References}

1. Boyett, M. R. \& Dobrzynski, H. The sinoatrial node is still setting the pace 100 years after its discovery. Circ. Res. 100, 1543-1545 (2007).

2. Mangoni, M. E. \& Nargeot, J. Genesis and regulation of the heart automaticity. Physiol. Rev. 88, 919-982 (2008).

3. Lakatta, E. G., Maltsev, V. A. \& Vinogradova, T. M. A coupled SYSTEM of intracellular $\mathrm{Ca} 2+$ clocks and surface membrane voltage clocks controls the timekeeping mechanism of the heart's pacemaker. Circ. Res. 106, 659-673 (2010).

4. Dobrzynski, H., Boyett, M. R. \& Anderson, R. H. New insights into pacemaker activity: promoting understanding of sick sinus syndrome. Circulation 115, 1921-1932 (2007).

5. Larson, E. D., Clair, J. R. S., Sumner, W. A., Bannister, R. A. \& Proenza, C. Depressed pacemaker activity of sinoatrial node myocytes contributes to the age-dependent decline in maximum heart rate. Proc. Natl Acad. Sci. USA 110, 18011-18016 (2013)

6. Vedantham, V., Galang, G., Evangelista, M., Deo, R. C. \& Srivastava, D. RNA sequencing of mouse sinoatrial node reveals an upstream regulatory role for Islet-1 in cardiac pacemaker cells. Circ. Res. 116, 797-803 (2015).

7. Shen, M. J. \& Zipes, D. P. Role of the autonomic nervous system in modulating cardiac arrhythmias. Circ. Res. 114, 1004-1021 (2014).

8. Schuessler, R. B., Boineau, J. P. \& Bromberg, B. I. Origin of the sinus impulse. J. Cardiovasc. Electrophysiol. 7, 263-274 (1996).

9. Murphy, C. \& Lazzara, R. Current concepts of anatomy and electrophysiology of the sinus node. J. Interv. Card. Electrophysiol. 46, 9-18 (2016).

10. Monfredi, O., Dobrzynski, H., Mondal, T., Boyett, M. R. \& Morris, G. M. The anatomy and physiology of the sinoatrial node-a contemporary review. Pacing Clin. Electrophysiol. 33, 1392-1406 (2010).

11. Linscheid, N. et al. Quantitative proteomics and single-nucleus transcriptomics of the sinus node elucidates the foundation of cardiac pacemaking. Nat. Commun. 10, 2889 (2019).

12. Picelli, S. et al. Full-length RNA-seq from single cells using Smart-seq2. Nat. Protoc. 9, 171-181 (2014).

13. Tang, F. et al. mRNA-Seq whole-transcriptome analysis of a single cell. Nat. Methods 6, 377-382 (2009).

14. Efimov, I. R., Fedorov, V. V., Joung, B. \& Lin, S. F. Mapping cardiac pacemaker circuits: methodological puzzles of the sinoatrial node optical mapping. Circ. Res. 106, 255-271 (2010).

15. Altomare, C. et al. Heteromeric HCN1-HCN4 channels: a comparison with native pacemaker channels from the rabbit sinoatrial node. J. Physiol. 549, 347-359 (2003).

16. Lukyanenko, $\mathrm{Y}$. O. et al. $\mathrm{Ca}(2+) /$ calmodulin-activated phosphodiesterase $1 \mathrm{~A}$ is highly expressed in rabbit cardiac sinoatrial nodal cells and regulates pacemaker function. J. Mol. Cell Cardiol. 98, 73-82 (2016).

17. Chandler, N. J. et al. Molecular architecture of the human sinus node: insights into the function of the cardiac pacemaker. Circulation 119, 1562-1575 (2009).
18. Marionneau, C. et al. Specific pattern of ionic channel gene expression associated with pacemaker activity in the mouse heart. J. Physiol. 562, 223-234 (2005).

19. Baruscotti, M. et al. Deep bradycardia and heart block caused by inducible cardiac-specific knockout of the pacemaker channel gene Hcn4. Proc. Natl Acad. Sci. USA 108, 1705-1710 (2011).

20. Stieber, J. et al. The hyperpolarization-activated channel HCN4 is required for the generation of pacemaker action potentials in the embryonic heart. Proc. Natl Acad. Sci. USA 100, 15235-15240 (2003).

21. Christel, C. J. et al. Distinct localization and modulation of Cav1.2 and Cav1.3 L-type Ca2+ channals in mouse sinoatrial node. J. Physiol. 590, 6327-6342 (2012).

22. Mangoni, M. E. et al. Functional role of L-type Cav1.3 Ca2+ channels in cardiac pacemaker activity. Proc. Natl Acad. Sci. USA 100, 5543-5548 (2003).

23. Platzer, J. et al. Congenital deafness and sinoatrial node dysfunction in mice lacking class D L-type Ca2+ channels. Cell 102, 89-97 (2000).

24. Baig, S. M. et al. Loss of $\mathrm{Ca}(\mathrm{v}) 1.3$ (CACNA1D) function in a human channelopathy with bradycardia and congenital deafness. Nat. Neurosci. 14, 77-84 (2011).

25. Shattock, $\mathrm{M}$. J. et al. $\mathrm{Na}+/ \mathrm{Ca} 2+$ exchange and $\mathrm{Na}+/ \mathrm{K}+$-ATPase in the heart. J. Physiol. 593, 1361-1382 (2015).

26. Bogdanov, K. Y., Vinogradova, T. M. \& Lakatta, E. G. Sinoatrial nodal cell ryanodine receptor and $\mathrm{Na}(+)-\mathrm{Ca}(2+)$ exchanger: molecular partners in pacemaker regulation. Circ. Res. 88, 1254-1258 (2001).

27. Torrente, A. G. et al. Burst pacemaker activity of the sinoatrial node in sodium-calcium exchanger knockout mice. Proc. Natl Acad. Sci. USA 112, 9769-9774 (2015)

28. Sakai, R., Hagiwara, N., Matsuda, N., Kassanuki, H. \& Hosoda, S. Sodium-potassium pump current in rabbit sino-atrial node cells. J. Physiol. 490, 51-62 (1996)

29. Vinogradova, T. M. et al. Sinoatrial node pacemaker activity requires $\mathrm{Ca}(2+) /$ calmodulin-dependent protein kinase II activation. Circ. Res. 87, 760-767 (2000).

30. Lisewski, U. et al. The tight junction protein CAR regulates cardiac conduction and cell-cell communication. J. Exp. Med. 205, 2369-2379 (2008).

31. Lim, B. K. et al. Coxsackievirus and adenovirus receptor (CAR) mediates atrioventricular-node function and connexin 45 localization in the murine heart. J. Clin. Invest. 118, 2758-2770 (2008).

32. Aziz, Q. et al. ATP-sensitive potassium channels in the sinoatrial node contribute to heart rate control and adaptation to hypoxia. J. Biol. Chem. 293 8912-8921 (2018).

33. Robinson, R. B. \& Siegelbaum, S. A. Hyperpolarization-activated cation currents: from molecules to physiological function. Annu. Rev. Physiol. 65 453-480 (2003)

34. Biel, M. et al. Hyperpolarization-activated cation channels: from genes to function. Physiol. Rev. 89, 847-885 (2009).

35. Lin, C. W. et al. VSNL1 co-expression networks in aging include calcium signaling, synaptic plasticity, and Alzheimer's disease pathways. Front. Psychiatry 6, 30 (2015).

36. van Eif, V. W. W. et al. Transcriptome analysis of mouse and human sinoatrial node cells reveals a conserved genetic program. Development 146, dev173161 (2019)

37. Matsa, E., Burridge, P. W. \& Wu, J. C. Human stem cells for modeling heart disease and for drug discovery. Sci. Transl. Med. 6, 239ps6 (2014).

38. Zhang, J. et al. Functional cardiomyocytes derived from human induced pluripotent stem cells. Circ. Res. 104, e30-e41 (2009).

39. Schweizer, P. A. et al. Subtype-specific differentiation of cardiac pacemaker cell clusters from human induced pluripotent stem cells. Stem Cell Res. Ther. 8, 229 (2017).

40. Ben-Ari, M. et al. From beat rate variability in induced pluripotent stem cellderived pacemaker cells to heart rate variability in human subjects. Heart Rhythm 11, 1808-1818 (2014).

41. Mandel, Y. et al. Human embryonic and induced pluripotent stem cell-derived cardiomyocytes exhibit beat rate variability and power-law behavior. Circulation 125, 883-893 (2012).

42. Chen, H., Zhang, A. \& Wu, J. C. Harnessing cell pluripotency for cardiovascular regenerative medicine. Nat. Biomed. Eng. 2, 392-398 (2018).

43. Morad, M. \& Zhang, X. H. Mechanisms of spontaneous pacing: sinoatrial nodal cells, neonatal cardiomyocytes, and human stem cell derived cardiomyocytes. Can. J. Physiol. Pharm. 95, 1100-1107 (2017).

44. Zhang, X. H. et al. Regionally diverse mitochondrial calcium signaling regulates spontaneous pacing in developing cardiomyocytes. Cell Calcium 57, 321-336 (2015).

45. Shafaattalab, S. et al. In vitro analyses of suspected arrhythmogenic thin filament variants as a cause of sudden cardiac death in infants. Proc. Natl Acad. Sci. USA 116, 6969-6974 (2019). 
46. Protze, S. I. et al. Sinoatrial node cardiomyocytes derived from human pluripotent cells function as a biological pacemaker. Nat. Biotechnol. 35, 56-68 (2017)

47. Fedorov, V. V., Glukhov, A. V. \& Chang, R. Conduction barriers and pathways of the sinoatrial pacemaker complex: their role in normal rhythm and atrial arrhythmias. Am. J. Physiol. Heart Circ. Physiol. 302, H1773-H1783 (2012).

48. Maltsev, V. A., Vinogradova, T. M. \& Lakatta, E. G. The emergence of a general theory of the initiation and strength of the heartbeat. J. Pharmacol. Sci. 100, 338-369 (2006).

49. Yang, Y. et al. Identification of a Kir3.4 mutation in congenital long QT syndrome. Am. J. Hum. Genet. 86, 872-880 (2010).

50. Wickman, K., Nemec, J., Gendler, S. J. \& Clapham, D. E. Abnormal heart rate regulation in GIRK4 knockout mice. Neuron 20, 103-114 (1988).

51. Gelling, R. W. et al. Lower blood glucose, hyperglucagonemia, and pancreatic alpha cell hyperplasia in glucagon receptor knockout mice. Proc. Natl Acad. Sci. USA 100, 1438-1443 (2003).

52. Baggio, L. L. et al. The autonomic nervous system and cardiac GLP-1 receptors control heart rate in mice. Mol. Metab. 6, 1339-1349 (2017).

53. Van Eif, V. W. W., Devalla, H. D., Boink, G. J. J. \& Christoffels, V. M. Transcriptional regulation of the cardiac conduction system. Nat. Rev. Cardiol. 15, 617-630 (2018).

54. Espinoza-Lewis, R. A. et al. Shox 2 is essential for the differentiation of cardiac pacemaker cells by repressing Nkx2-5. Dev. Biol. 327, 378-385 (2009).

55. Blaschke, R. J. et al. Targeted mutation reveals essential functions of the homeodomain transcription factor Shox 2 in sinoatrial and pacemaking development. Circulation 115, 1830-1838 (2007).

56. Rosen, M. R. Gene therapy and biological pacing. N. Engl. J. Med. 371, 1158-1159 (2014).

57. Verheijck, E. E. et al. Distribution of atrial and nodal cells within the rabbit sinoatrial node: models of sinoatrial transition. Circulation 97, 1623-1631 (1998).

58. Sharpe, E. J., Clair, J. R. S. \& Proenza, C. Methods for the isolation, culture, and functional characterization of sinoatrial node myocytes from adult mice. $J$. Vis. Exp. 116, e54555 (2016).

59. Köhncke, C. et al. Isolation and $\mathrm{Kv}$ channel recordings in murine atrial and ventricular cardiomyocytes. J. Vis. Exp. 12, e50145 (2013).

60. $\mathrm{Hu}, \mathrm{Y}$. J. et al. Simultaneous profiling of transcriptome and DNA methylome from a single cell. Genome Biol. 17, 88 (2016).

61. Picelli, S. Full-length single-cell RNA sequencing with smart-seq2. Methods Mol. Biol. 1979, 25-44 (2019).

62. Kim, D., Langmead, B. \& Salzberg, S. L. HISAT: a fast spliced aligner with low memory requirements. Nat. Methods 12, 357-360 (2015).

63. Anders, S., Pyl, P. T. \& Huber, W. HTSeq-a python framework to work with high-throughput sequencing data. Bioinformatics 31, 166-169 (2015).

64. Hyun, S. W. et al. The assessment of electrophysiological activity in humaninduced pluripotent stem cell-derived cardiomyocytes exposed to dimethyl sulfoxide and ethanol by manual patch clamp and multi-electrode array system. J. Pharmacol. Toxicol. Met 87, 93-98 (2017).

65. Liu, W. et al. MG53, a novel regulator of KChIP2 and $\mathrm{I}_{\text {to,f }}$, plays a critical role in electrophysiological remodeling in cardiac hypertrophy. Circulation 139, 2142-2156 (2019).

66. Vettel, C. et al. Phosphodiesterase 2 protects against catecholamine-induced arrhythmia and preserves contractile function after myocardial infarction. Circ. Res. 120, 120-132 (2017).

67. Fenske, S. et al. Comprehensive multilevel in vivo and in vitro analysis of heart rate fluctuations in mice by ECG telemetry and electrophysiology. Nat. Protoc. 11, 61-86 (2015).

\section{Acknowledgements}

We thank Dr. E.R. Roy for the language editing of the manuscript. This work was funded by the the Grants from the National Key Research and Development Plan (2019YFA0801501 to Y.-H. Chen; 2017YFC1001301 to Z.X.), the Key Program of National Natural Science Foundation of China (82088101, 81530017 and 81930013 to Y.-H.C.), the Programs of the National Natural Science Foundation of China (81770397 to Y.-H.C.; 81430026 to Z.X.; 81770267 and 82070271 to D.L.), the Program of National Key Research and Development Plan (2016YFC1000208 to Z.X.), the Program of Science and Technology Commission of Shanghai Municipality (16JC1404700 to Z.X.), the Grants from China Postdoctoral Science Foundation (2017M611623, to Jinfeng Xue). Key Disciplines Group Construction Project of Pudong Health Bureau of Shanghai (PWZxq2017-05), Top-level Clinical Discipline Project of Shanghai Pudong District (PWYgf2018-02), Research Unit of Origin and Regulation of Heart Rhythm, Chinese Academy of Medical Sciences (2019RU045). Y.-H.C. is a Fellow at the Collaborative Innovation Center For Cardiovascular Disease Translational Medicine, Nanjing Medical University.

\section{Author contributions}

D.L. carried out experiments, analyzed data, and wrote the manuscript. J.X. performed bioinformatics analysis and wrote the manuscript. L.G. and L.Z. performed sinoatrial node cells isolation and data analysis. K.X., H.Z., D.X., F.Z., J.L., Y.L., and L.L. carried out experiments. B.L. and Q.Z. constructed the single-cell library and performed data analysis. J.Y. provided important revisions in the manuscript. Z.X. funded the projects, participated in discussions, and analyzed data. Y.-H.C. designed, led and funded the projects, and wrote the manuscript.

\section{Competing interests}

The authors declare no competing interests.

\section{Additional information}

Supplementary information is available for this paper at https://doi.org/10.1038/s41467020-20448-x.

Correspondence and requests for materials should be addressed to Z.X. or Y.-H.C.

Peer review information Nature Communications thanks the anonymous reviewer(s) for their contribution to the peer review of this work.

Reprints and permission information is available at http://www.nature.com/reprints

Publisher's note Springer Nature remains neutral with regard to jurisdictional claims in published maps and institutional affiliations.

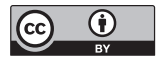

Open Access This article is licensed under a Creative Commons Attribution 4.0 International License, which permits use, sharing, adaptation, distribution and reproduction in any medium or format, as long as you give appropriate credit to the original author(s) and the source, provide a link to the Creative Commons license, and indicate if changes were made. The images or other third party material in this article are included in the article's Creative Commons license, unless indicated otherwise in a credit line to the material. If material is not included in the article's Creative Commons license and your intended use is not permitted by statutory regulation or exceeds the permitted use, you will need to obtain permission directly from the copyright holder. To view a copy of this license, visit http://creativecommons.org/ licenses/by/4.0/.

(C) The Author(s) 2021 